\title{
Rechtsgeschichte Legal History
}

www.rg.mpg.de

http://www.rg-rechtsgeschichte.de/rg25

Zitiervorschlag: Rechtsgeschichte - Legal History Rg 25 (2017)

$\operatorname{Rg} 252017$

$162-182$

http://dx.doi.org/10.12946/rg25/162-182

\section{Daniel Damler*}

\section{Synästhetische Normativität}

[Synaesthetic Normativity]

* Max-Planck-Institut für europäische Rechtsgeschichte, Frankfurt am Main, damler@rg.mpg.de

Dieser Beitrag steht unter einer Creative Commons cc-by-nc-nd 3.0 


\begin{abstract}
Values regulate and determine normative orders. According to a widespread assumption, values arise for their parts solely within the borders of the specific normative order (aesthetics, science, ethics, law etc.) they are intended for. The article challenges this view, arguing that aesthetic, epistemic and moral (legal, political) values are correlated because of a common underlying mechanism. The »synaesthesia of values" facilitates a constant exchange of principles and concepts between apparently distinct normative spheres.
\end{abstract}

Keywords: kalokagathia, category mistake, metaphor, values, attractiveness stereotype 


\section{Daniel Damler Synästhetische Normativität}

I.

Im Herzen Frankreichs, südlich von Paris und Orléans, östlich von Blois, in einem ausgedehnten Waldgebiet liegt das Schloss von Chambord, das prächtige, das unvergleichliche Chambord, Prunkund Jagdresidenz Franz' I., architektonischer Höhe- und Wendepunkt der französischen Renaissance. Karl V., Kaiser des Heiligen Römischen Reiches und des allerchristlichsten Königs ewiger Rivale, soll das Schloss, als er es 1539 zu Gesicht bekam, gepriesen haben als den »Inbegriff dessen, was menschliche Kunst vermag. « ${ }^{\mathbf{1}}$

Darüber lässt sich streiten. Aber gewiss ist Chambord der Inbegriff dessen, was menschliche Kunst nach Ansicht frühneuzeitlicher Fürsten vermögen soll, nämlich ästhetische Affekte in den Dienst der Macht, in den Dienst einer politischen Agenda zu stellen. Kein anderer Palast jener Zeit dokumentiert den Herrschaftsanspruch seines Bauherren auf eine vergleichbar ingeniöse Weise wie die prachtvolle maison de plaisance an der Loire. Das Gebäude ist nicht wie üblich von innen nach außen, sondern von außen nach innen konzipiert. Der zentrale Bauteil, der Donjon, besteht aus vier Wohntürmen, die auf einem quadratischen Grundriss über eine zweiläufige Wendeltreppe in der Mitte des Bauwerks verbunden sind (Abb.). ${ }^{2}$ Nicht die Fassade, der imposante escalier à double révolution im Inneren ist zeremonieller Dreh- und Angelpunkt des baulichen Arrangements. Der Treppenturm durchstößt das Dach und geht über in ein lichtdurchflutetes, von einer steinernen Bügelkrone gerahmtes Kuppelgewölbe. Den Abschluss des Lichtturms bildet eine Laterne, geschmückt mit den französischen Lilien.

Wer Chambord betritt, den verschlingt also ein gigantischer dreidimensionaler Wirbel: Aus der Peripherie gelangt er in das Zentrum, von der Erde in den Himmel, aus dem Dunkel in die Helligkeit. Der Besucher erlebt am eigenen Leib die Wirkung dreier binärer elementarästhetischer Qualitäten (mittig/peripher, oben/unten, hell/ dunkel), denen jeweils ein positiver und ein negativer Wert zugewiesen ist. ${ }^{3}$ Der Nähe zur Lilienkrone entspricht die ästhetische Wertigkeit. Doch sind solche ästhetischen Wertigkeiten in einem Gebäude, das der Inszenierung legitimer Herrschaft dient, nicht Selbstzweck, sondern Mittel zum Zweck. Die verwegene Konstruktion ergibt nur Sinn und rechtfertigt nur dann die ungeheuren Investitionen, wenn man sie als ein Instrument begreift, das auf die subtile, aber gerade deshalb umso eindringlichere Vergegenwärtigung traditioneller Herrschertugenden wie Güte, Gerechtigkeit und Weisheit zielt. Das Verfahren kommt ohne schriftliche Anleitungen, ja selbst ohne allegorische Darstellungen aus. Lediglich für die Präsenz der königlichen Herrschaftszeichen im Treppenturm - die Imprese Franz' I., der gekrönte Salamander, und seine Initiale »F« - wurde Sorge getragen, um die korrekte Zuordnung der Tugenden zu gewährleisten. ${ }^{4}$ Auch erfuhr die Lichtmetaphorik eine gewisse Verstärkung durch das Anbringen korrespondierender Emblemata im Kuppelgewölbe (Flammenschalen, Fackeln, Kandelaber usw.). ${ }^{5}$

Von der sich in Chambord seit fünfhundert Jahren tagtäglich aufs Neue vollziehenden Koppelung von Werturteilen, dem »Zugleich-Wahrnehmen«, dem »Mit-Empfinden« ästhetischer, moralischer und epistemischer Qualitäten, soll im Folgenden die Rede sein. Im Unterschied zur Simultanstimulation verschiedener Sinneswahrnehmungen (z. B. Töne und Farben) durch einen einzigen Sinnesreiz - Synästhesie im eigentlichen Sinn (dazu III. s.v. Synästhesie / cross-modale Korrespondenz) handelt es sich bei der "normativen Synästhesie» nicht um eine seltene Abweichung von einem als Standard definierten Zustand, sondern um einen alltäglichen Vorgang, um eine anthropologische Konstante.
1 Metternich (1985) 23.

2 Aus: Jean Guillaume, Comprendre Chambord, Monuments historiques: dossier technique, no. 2 (1983). Herrn Guillaume danke ich herzlich für seine Genehmigung.
3 Vgl. Prinz/Kecks (1994) $278 \mathrm{f}$.

4 Prinz (1980) 26; Prinz/Kecks (1994) 278.

5 Prinz (1980) 26; Prinz/Kecks (1994) 279. 
Lässt sich das Phänomen selbst ebenso gut anhand einer Vielzahl anderer Gebäude, aber auch anhand von Kleidungsstücken oder musikalischen Darbietungen vorführen, so liegt der besondere Reiz Chambords darin, dass die Architektur des Schlosses zugleich das Modell oder jedenfalls ein denkbares Modell des für die normative Synästhesie verantwortlichen kognitiven Mechanismus bereitstellt. Um das Konstruktionsprinzip besser zu verstehen, das Chambord auszeichnet, ist es hilfreich, sich ein wenig mit dem mutmaßlichen Spiritus rector des Vorhabens zu beschäftigen. Gemeint ist nicht Franz I., obwohl keine Zweifel bestehen, dass er sich persönlich engagierte und regen Anteil nahm an der künstlerischen Gestaltung. Ohne sachverständigen Beistand konnte indes auch ein so kultivierter Herrscher wie der RoiChevalier ein Großprojekt dieses Ausmaßes nicht realisieren. Wer den König beraten, wer für ihn den ungewöhnlichen Grundriss entworfen hat, ist bis heute nicht abschließend geklärt und wird sich wohl nie mit letzter Sicherheit erweisen lassen, da es an Unterlagen aus der Planungsphase fehlt. ${ }^{\mathbf{6}}$

Dass es Leonardo da Vinci war, der wichtige Ideen zur architektonischen Konzeption Chambords beisteuerte, gilt aber heute als wahrscheinlich. ${ }^{7}$ Zwar wird man ihn nicht schlechthin als »den« Architekten Chambords bezeichnen kön-

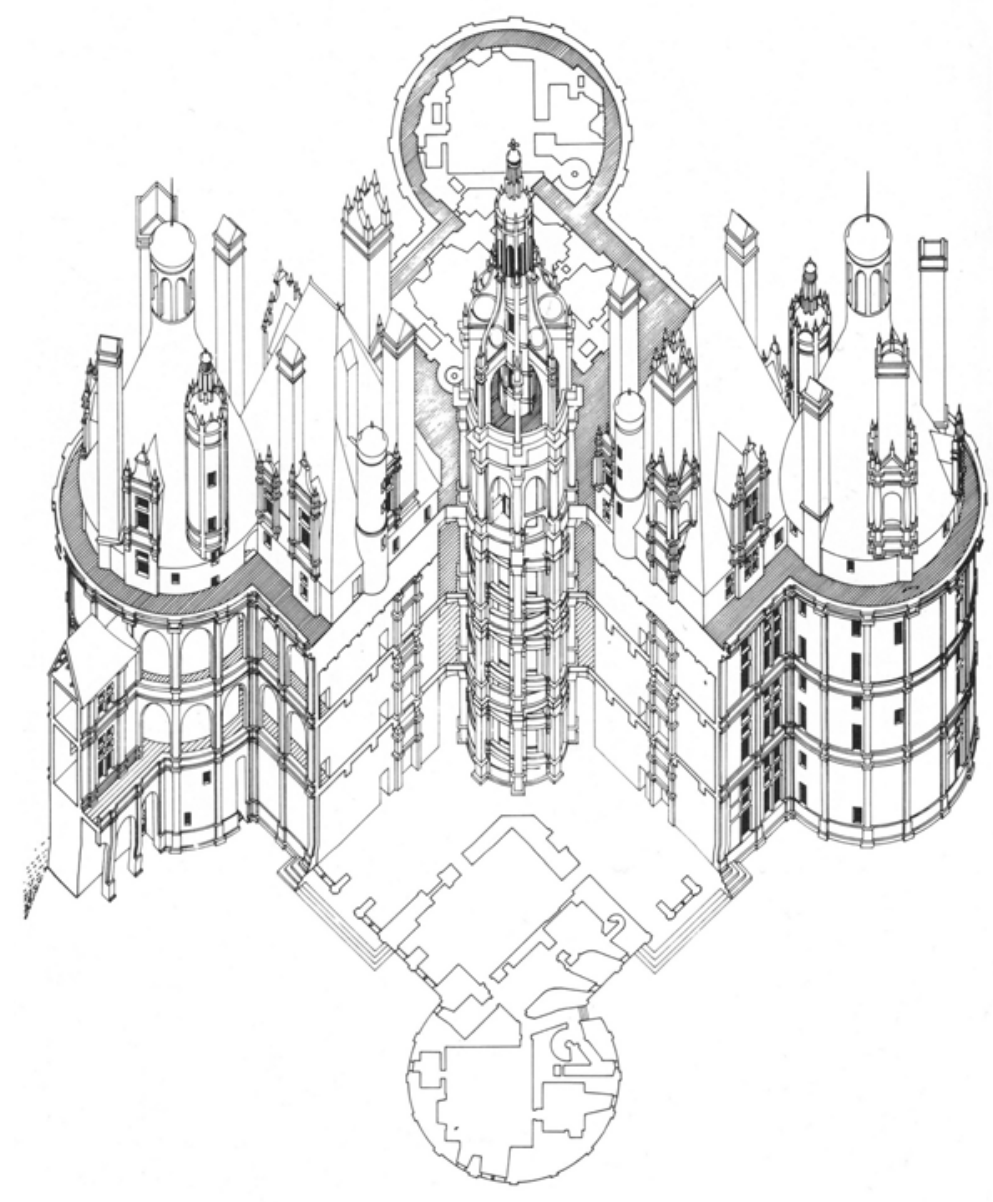

6 Vgl. Guillaume (2009) 123;

MeTternich (1985) 94-107.

7 Grundlegend Guillaume (1974);

Heydenreich (1952); MetTernich

(1985) $101 \mathrm{f}$. 
nen, da er bereits im Mai 1519 verstorben war, wenige Monate vor Beginn der Bauarbeiten, doch das schließt nicht aus, dass ein erster Entwurf aus seiner Feder stammt. Immerhin residierte der Italiener seit 1516 in der Nähe des in Aussicht genommenen Standortes - im Schloss Clos Lucé, südwestlich von Blois - und erfreute sich der höchsten Wertschätzung des jungen Monarchen. Auf einen Gedankenaustausch mit seinem renommiertesten Berater wird Franz I. wohl kaum verzichtet haben. ${ }^{\mathbf{8}}$

Überdies gibt es auffällige konzeptionelle Überschneidungen und Korrespondenzen, die für eine Beteiligung Leonardo da Vincis sprechen. Die für die Zeit und allemal für Frankreich untypische Idee eines Zentralbaus sowie einer in der Mitte positionierten mehrläufigen Treppe lassen sich in zahlreichen seiner Skizzen nachweisen. ${ }^{9}$ Insbesondere der Einfall, alle Etagen der vier Wohntürme über eine einzige Wendeltreppe zugänglich zu machen, entspricht vollkommen Leonardos Ideal effizienter Material- und Raumnutzung. Der »Vater der Bionik« hat sich wie kein anderer darauf verstanden, die für biologische Systeme charakteristische Multifunktionalität und überlegene Ressourcenallokation auf artifizielle Verfahren und Konstruktionen zu übertragen. ${ }^{10}$ In Leonardos Kosmos war der Gedanke einer um sich selbst kreisenden Zentralachse, die alles in sich aufnahm und alles aus sich heraus bewegte, eine Art Leitmotiv von der Luftschraube, dem berühmten helix pteron, und den Mühlenrädern bis hin zu den Strudel- und Wirbelskizzen in den Sturm- und Wasserstudien seiner späten Jahre.

Der italienische Universalgelehrte war indes nicht blind für die Schwächen der Ein-TreppenLösung. Der Nachteil bestand namentlich darin, dass sich die Wahrscheinlichkeit einer Begegnung und Kommunikation zwischen den Bewohnern der vier Türme dramatisch erhöhte, denn sie alle hatten bis kurz vor Erreichen ihrer Behausung den gleichen Weg zurückzulegen, während sich bei einem Gebäude mit vier separaten seitlichen Zugängen weniger Gelegenheiten boten, absichtlich oder versehentlich andere Räume als die zugewiesenen zu Gesicht zu bekommen. In vielen
Fällen mögen ein kommunikatives Zentrum und eine fortwährende Grenzüberschreitung unschädlich oder sogar erwünscht sein. Es gibt aber auch Fälle, in denen die Nutzungsart des Gebäudes sich mit dem Prinzip der offenen, kommunikativen Mitte nur schwer vereinbaren lässt. So kann es aus sicherheitspolitischen Erwägungen empfehlenswert sein, militärische Einheiten auf verschiedene Stützpunkte oder Gebäude zu verteilen, anstatt sie in einer einzigen großen Kaserne unterzubringen. Die Vorteile effizienten Bauens wiegen in der Militärarchitektur weniger schwer als handfeste Bedrohungen für die innere Sicherheit durch Revolten und Aufstände. ${ }^{\mathbf{1 1}}$

Leonardo da Vinci schien sich dessen bewusst gewesen zu sein, als er in seiner Mailänder Periode, also zu einer Zeit intensiver Beschäftigung mit Zweck- und Militärbauten, eine vierläufige Zentraltreppe zu Papier brachte, die es nicht erlaubte, von einem Treppenlauf in den anderen zu gelangen. ${ }^{12}$ Andrea Palladio, der irrtümlich glaubte, dieses Modell sei auf Anraten Leonardos in Chambord realisiert worden, beschrieb in seinen »Quattro Libri« (Venedig 1570) die Konstruktion wie folgt: »Es handelt sich um vier Treppen mit vier Eingängen, je ein Eingang gehört zu einer Treppe. Je eine Treppe führt über und parallel zu einer anderen hinauf, und dies in der Art, dass die Treppen jeweils, wenn man sie in die Mitte des Gebäudes setzt, vier separaten Raumfolgen dienen, ohne dass die Personen, die in einer Wohnung wohnen, über die Treppe der anderen Wohnung gehen müssen. « ${ }^{\mathbf{1 3}}$

Moderne Berechnungen haben ergeben, dass eine vierläufige Treppe den gesamten Kern des Donjons ausgefüllt und die Raum- und Materialersparnis, die ein Zentralbau mit sich bringt, zu einem guten Teil wieder zunichte gemacht hätte ganz abgesehen von der unvorteilhaften ästhetischen Wirkung eines so wuchtigen Treppenturms. ${ }^{14}$ Für ein Jagdschloss, das eher auf Inklusion als auf Fragmentierung der Jagdgesellschaft zielte, wäre der Aufwand in jedem Fall völlig unangemessen gewesen.

Für unser Thema sind gleichwohl beide Aspekte wichtig: die Effizienzdividende und die von Leo-
8 Guillaume (2009) 123; Metternich (1985) 102.

9 Metternich (1985) 103.

10 Vgl. Nachtigall (1997) 9, 21-24.

11 Guillaume (2009) 124.
12 Dazu Guillaume (2009) 124; Liesen (1999) 107; MetTernich (1985) 91.

13 Palladio (1983) 105.

14 Metternich (1985) 91 f.; Liesen (1999) $126 \mathrm{f}$. 
nardo durch seinen Mailänder Entwurf indirekt bestätigten Ordnungsdefizite einer offenen Zentraltreppe. Es ist einiges gewonnen, wenn wir uns den Mechanismus, der der normativen Synästhesie zu Grunde liegt, als einen solchen in der Mitte positionierten, den Bewohnern aller Gebäudeteile zugänglichen Aufgang vorstellen. So verstehen wir besser, warum einerseits Wertungen in unserem Leben so ungeheuer präsent und andererseits nur mit einem gewissen gedanklichen Aufwand voneinander zu unterscheiden sind. Wer sich beim Treppensteigen nicht konzentriert, der nimmt leicht den falschen Ausgang und befindet sich dann unversehens in einem Teil des Schlosses, in dem er nichts zu suchen hat.

In einem Vortrag aus dem Jahr 1952 (»Visual Metaphors of Value in Art «) hat Ernst Gombrich die Eigenheiten kongruenter Wert-Erfahrungen hellsichtig beschrieben: "Wesentlich ist, dass in unserem Erleben immer Resonanzen aus anderen Wertsystemen mitschwingen. Es gab einst gute Gründe, gegen eine Verwirrung der Werte in der Kunstkritik zu protestieren, vor allem gegen eine oberflächliche Verwechslung von Kunst und Moral. Aber heute, wo diese Gefahr nicht mehr besteht, sollten wir ohne Zögern die Tatsache anerkennen, dass es wenige Menschen gibt, die Kunst niemals als sittlichen Wert erleben. " 15

Ein Kunstsachverständiger, der einem Künstler vorschreiben wollte, auf welche Weise er Wertsymbole hervorbringen müsse, mache sich lächerlich. Und dennoch: „Die wahren oder vermeintlichen Werte, die der Kritiker aus den Parolen der jüngsten sozialen Strömungen herauszuhören glaubt und die er dann selbst schlagwortartig weiter in Umlauf setzt, umschwirren den schaffenden Künstler von allen Seiten; im Positiven und im Negativen bestimmen sie nicht selten die Richtung, die er nimmt. Umso wichtiger ist es, sich darüber im Klaren zu sein, dass die Metaphern der Kunst Metaphern sind, dass sie aber ihren Ursprung haben in jenem innersten Wesen, wo das `Gute<, das >Reine $<$, das `Edle $<$, das >Wahre , das `Gesunde und Natürliche` und das `Echte und Ehrliche` nur verschiedene Aspekte eines einzigen unaussprechlichen Erlebnisses sind, des Erlebnisses von Wert schlechthin, das zum ganzen Menschen spricht. « ${ }^{\mathbf{1 6}}$ Dass bereits Kant Interesse an der sittlich-ästhetischen "Grenzüberschreitung “ ${ }^{17}$ bekundet hatte, wird Gombrich vermutlich in seiner Auffassung bestärkt haben. »Wir benennen«, heißt es in der »Kritik der Urteilskraft«, »schöne Gegenstände der Natur, oder der Kunst, oft mit Namen, die eine sittliche Beurtheilung zu Grunde zu legen scheinen. Wir nennen Gebäude oder Bäume majestätisch und prächtig, oder Gefilde lachend und fröhlich; selbst Farben werden unschuldig, bescheiden, zärtlich genannt, weil sie Empfindungen erregen, die etwas mit dem Bewusstsein eines durch moralische Urteile bewirkten Gemüthszustandes Analogisches enthalten. « ${ }^{\mathbf{1 8}}$

Zwei Jahre nach Gombrich - 1954 - veröffentlichte Erwin Panofsky eine Studie, die sich wie ein Kommentar zu dessen Ausführungen liest und die Relevanz normativer Synästhesien an einem konkreten Beispiel veranschaulicht. In »Galileo as a Critic of the Arts« befasst sich Panofsky mit Galileo Galileis Unfähigkeit, das Erste Keplersche Gesetz als wahr anzuerkennen, ein Umstand, der die Verehrer des Italieners bis heute irritiert und verstört. ${ }^{19}$ Mit großer Wahrscheinlich kannte Galilei die (bereits 1609 publizierte) These Keplers, dass sich die Planeten nicht auf kreisförmigen (wie von Kopernikus angenommen), sondern auf elliptischen Bahnen bewegen. Früh akzeptierte Galileis unmittelbares Umfeld die wissenschaftliche Überlegenheit des neuen Modells - nur eben der Meister selbst nicht. ${ }^{20}$ Dass Galilei auf dem privilegierten Status des Kreises beharrte, stand - wie Panofsky zeigt - in Einklang mit der antiken Überlieferung und zudem mit dem ästhetischen Programm der Hochrenaissance. Die Ellipse - als ein »verunstalteter Kreis « - passte nicht in das Formenrepertoire einer Zeit, die das Schöne und Erhabene zur Ansicht bringen wollte. Die frühesten Darstellungen der Ellipse finden sich in der Plastik erst bei Pierino da Vinci und Guglielmo della Porta, in der Architektur nicht vor Baldassare Peruzzi. ${ }^{21}$
15 Gombrich (1973) 35.

16 Gombrich (1973) 56 (Hervorhebung im Original).

17 Vgl. Hartmann (1949) 92.

18 Kant (1968) 462 f. ( $\left.\int 59\right)$.

19 »Dass in Galileos Lebenswerk dieser entscheidende Fortschritt keine Spu- ren hinterlassen hatte, ist ein groteskes Beispiel dafür, dass schöpferische Menschen oft nicht rezeptiv orientiert sind « - EINSTEIN (1953) XVI, zitiert nach PANOFSKY (1954) 24.

20 PANofsky (1954) $22 \mathrm{f}$.

21 Panofsky (1954) 25-28. 
Demnach unterschlug der künstlerisch begabte und empfindsame Galilei ein Konzept, das ihn als Wissenschaftler hätte überzeugen müssen, allein oder auch deshalb, weil es sich mit seiner ästhetischen Prägung nicht vertrug. Das Erste Keplersche Gesetz war hässlich, aber wahr.

II.

Das Phänomen, das Gombrich und Panofsky aus kunsthistorischer Perspektive mit Blick auf die künstlerische und wissenschaftliche Praxis skizzieren, geht auch den Juristen etwas an, ja die Jurisprudenz als Normenwissenschaft par excellence muss den »Resonanzen aus anderen Wertsystemen«, muss dem »einzigen unaussprechlichen Erlebnis, dem Erlebnis von Wert schlechthin« eine noch größere Aufmerksamkeit entgegenbringen als die Kunsttheorie. Folgende Thesen, die verschiedene Erklärungsansätze zusammenführen, stehen zur Diskussion:

1. Es gibt keine »schöne Wissenschaft, sondern nur schöne Kunst «. ${ }^{\mathbf{2 2}}$ Und schönes Recht gibt es schon gar nicht. An der kategorialen Unterscheidung zwischen den verschiedenen normativen Ordnungen ist unbedingt festzuhalten. Als Ideal, als Leitbild, als »regulative Idee ${ }^{23}$ die unser Handeln und Denken bestimmen soll, hat sie ihre Berechtigung.

2. Davon zu unterscheiden ist die Frage, inwieweit dem Ideal die Wirklichkeit entspricht und ob nicht gerade die Beobachtung und Beschreibung unseres Unvermögens, sich ausnahmslos innerhalb der durch die binäre Codierung "gut/böse«, "wahr/unwahr«, "schön/hässlich « usw. gezogenen Grenzen zu bewegen, Gegenstand wissenschaftlicher Untersuchung sein muss. Der Fall Galilei führt vor Augen, dass die Wertungskorrespondenz »hässlich/unwahr« die kategorienadäquate Urteilsfindung zu unterlaufen vermag. Jedenfalls in ihrer populären Ausprägung und Verkürzung hat die Systemtheorie der Fehlvorstellung
Vorschub geleistet, in Systemen bestehe ein "unaufhebbares Fürsichsein «, ${ }^{\mathbf{2 4}}$ eine »operative Geschlossenheit " ${ }^{25}$ in dem Sinne, dass die Abgrenzung zwischen System und Umwelt nach Art einer automatisierten Rechenoperation fehlerfrei funktioniere. $^{\mathbf{2 6}}$ Der rigide Schematismus kybernetischer Modelle hat den Blick verstellt auf die »Kontaktzonen «, die »Übergänge in eine Anderwelt «. ${ }^{27}$

3. Ausgehend von der Beobachtung Kants, dass »schöne Gegenstände« in uns »Empfindungen erregen, die etwas mit dem Bewusstsein eines durch moralische Urteile bewirkten Gemütszustandes Analogisches enthalten «, empfiehlt es sich, die Existenz eines einheitlichen Evaluationsmechanismus zu unterstellen: Wertungen werden über zeitlich vorauslaufende Affekte reguliert. Die Zuordnung zu einer bestimmten Normenkategorie ist eine analytische Leistung, die erst in einem zweiten Schritt erfolgt. Aufgrund der partiellen Identität der involvierten Abläufe sind "analogische« Interferenzen unvermeidbar. Der geteilten Nutzung entspricht ein Mit-Erleben, ein Verkoppeln moralischer, ästhetischer und epistemischer Wertungen. Dieser Effekt hat möglicherweise sowohl eine phylogenetische als auch eine ontogenetische Dimension. Die »Integration von aufeinanderfolgenden Strukturen, von denen jede die nächste aufbaut« (Piaget), ${ }^{\mathbf{2 8}}$ ist in der stammesgeschichtlichen Entwicklung ebenso zu beobachten wie in der Individualentwicklung. Elementare Urteile über Umweltgefahren oder das Verhalten der Artgenossen ${ }^{29}$ dienen als Muster für das Bewerten komplexer gesellschaftlicher und natürlicher Vorgänge und Zustände.

4. Wir bewerten unsere Umwelt fortwährend und fortwährend richten wir unser Handeln nach Wertungen aus. Sie sind die Zentralachse unseres Daseins, ein gigantischer Regulator und rotierender Riesenscheinwerfer, der die Nacht um uns durch sein wanderndes Schlaglicht erhellt. »Das Werten durchzieht das ganze geistige Leben; es führt zur psychischen Auslese der Bewusstseinsinhalte ...; es leitet das Denken und Erkennen, es
22 Kant (1968) 403 ( $(44)$ : »Wortverwechselung«.

23 Kant (1781) A 671. Dazu DörflinGER/ KRUCK (2011); FERRARI (1998) 491-523; Höfre (2011) insbes. 272-277.

24 Luhmann (1997) 107.

25 Vgl. Luhmann (1993) 38-123. Dazu Vesting (2015) Rn. 112-128.
26 Vgl. auch die Kritik von Vesting (2017) 242-248; Vesting (2015) Rn. 127 f.; eingehend und kritisch zu den Modellannahmen des systemtheoretischen Gesellschaftsbildes Lepsius (1999) 35-51.

27 Im Anschluss an Vesting (2017) 245 und Koschorke (2012) $120 \mathrm{f}$.

28 Piaget/Inhelder (1996) 151.
29 Vgl. Todorov/Baron/OosterhoF (2008); TODOROV/SAID / ENGELL/ OOSTERHOF (2008). 
wirkt in der Wissenschaft, in der Weltanschauung. Alles Handeln ist direkt oder indirekt Ausdruck von Wertungen - das theoretische wie das praktische Handeln. ${ }^{30}$ Verglichen mit der großen Zahl an Werturteilen kommt es selten zu Missgriffen ${ }^{31}$ mit ernsten Folgen. Vor allem bei kurzfristigen und nebensächlichen Entscheidungen können Friktionen auftreten (dazu III. s.v. Raum-, Hygiene-, Klang-, Proportions-»Werte«). Je mehr Zeit zur Verfügung steht und je höher die Konzentration, desto höher ist auch die Wahrscheinlichkeit, dass Fehlschlüsse rechtzeitig erkannt und selbständig korrigiert werden.

5. Erst recht können Irrtümer, die Einzelpersonen in Alltagssituationen unterlaufen, nicht einfach auf überindividuelle Prozesse hochgerechnet werden. Kollektive normative Kategorienfehler ${ }^{32}$ haben zur Voraussetzung, dass nicht nur die Eigenkontrolle versagt, sondern auch die üblichen Rationalisierungseffekte durch kommunikatives Handeln ausbleiben, die sich einstellen, wenn Teilnehmer eines (herrschaftsfreien) Diskurses Argumente austauschen. In den modernen Technikund Naturwissenschaften sind solche Fehler selten. Ein wenig anders verhält es sich mit gesellschafts-, wirtschafts- und rechtspolitischen Diskursen. Da oft schon keine Einigkeit über die einzuhaltenden Standards besteht, Rückkoppelungen in sozialen Systemen in der Regel nicht zeitnah erfolgen und Gesellschaften (Wirtschafts-, Rechtsordnungen) zu unübersichtlich und beziehungsreich sind, um Ursache und Wirkung eindeutig zuzuordnen, ergeben sich Spielräume für epidemische kognitive Verzerrungen.

6. Ob normative Kategorienfehler auf Akzeptanz stoßen und tradiert werden, hängt von einer Reihe gesellschaftlicher und kultureller Faktoren ab. In Betracht kommen etwa die Rezeption bestimmter philosophischer Traditionen, die - wie der Platonismus - für wertungsmonistische Ansätze empfänglich sind (dazu III. s.v. Kalokagathie), oder kulturtechnische Innovationen wie zum Beispiel die Erfindung des Films (dazu III. s. v. Montage der Attraktionen). Die Stabilität eines Wertekanons spielt ebenfalls eine große Rolle. In Umbruchsund Krisenzeit steigt die Wahrscheinlichkeit, dass Leitbilder, die anderen, von einer Denaturierung nicht betroffenen Normenkategorien angehören, an die Stelle der zeitweise dysfunktionalen Werte treten. Mit Hilfe solcher Surrogate lässt sich die Illusion eines Konsenses erzeugen, ohne dass je eine Auseinandersetzung über Inhalte stattgefunden hätte. Auf die Weise können etwa ästhetische "Tugenden« die Funktionen moralischer Verhaltensmaßstäbe übernehmen und deren schwindende Verbindlichkeit kompensieren (dazu III. s.v. Konsumismus / Sachkultur).

7. Normative Kategorienfehler können in ganz unterschiedlicher Gestalt in Erscheinung treten. Das Spektrum reicht von fehlerhaften Parallelwertungen, zum Beispiel beim Betrachten eines menschlichen Gesichtes (dazu III. s.v. Attraktivitätsstereotyp), einer höfischen Residenz oder einer zeremoniellen Handlung, bis hin zu komplexeren Vorgängen wie der Diffusion kategorienfremder Schlüsselbegriffe. Solche Grenzüberschreitungen sind deshalb vielschichtige Erscheinungen, weil die diffundierenden Begriffe, Parolen, Lehrsätze, Theoriefragmente, Denkfiguren usw. erst »lesbar« gemacht, an die empfangende Normenkategorie assimiliert und in konkrete Handlungsanleitungen übersetzt werden müssen. Der Translationsprozess geht mit einer »Rationalisierung « einher, d.h. es findet ein Abgleich mit der Realität sowie den kategorienadäquaten Maßstäben und Argumenten statt (dazu III. s.v. Norm/normatives Implantat). Dennoch bleibt in der Regel ein Restbestand an nicht sachgerechten Bedeutungsgehalten erhalten. Insoweit sind normative Grenzüberschreitungen »fehlerhaft", was nicht ausschließt, dass sie nach Art produktiver Missverständnisse in Einzelfällen sinnvolle Innovationen anstoßen können.

8. Auf der gleichen (hohen oder niedrigen) Wertungsebene gibt es keine Übertragungsrichtung, die a limine ausgeschlossen ist. Die Neigung, dem Bereich des äußeren, »ästhetischen« Gefallens zuzuordnende Inhalte zu übernehmen und zu assimilieren, mag aufgrund unserer Disposition
30 EISLER/Roretz (1930) Art. »Wert « 514.

31 Sofern hier und im Folgenden von »Missgriffen «, »Fehlern«, »Fehlschlüssen«, »Irrtümern« usw. die Rede ist, beziehen sich diese Begriffe auf die Ergebnisse »analogischen« Denkens und Empfindens. Eine darüber hinausgehende Bewertung, gar eine moralisierende Geschichtskritik ist damit nicht verbunden.

32 Der Ausdruck »category-mistake« geht auf Gilbert Ryle zurück oder wurde jedenfalls durch ihn populär: Ryle (1949) 16. 
als sinnliche Wesen besonders ausgeprägt sein (dazu III. s.v. Analoges Denken / Metapher), doch kommen auch gänzlich anders geartete Rezeptionsvorgänge in Betracht, etwa wenn populäre (natur-)wissenschaftliche Dogmen, die an sich keine Geltung jenseits der Kategorie "wahr - unwahr» beanspruchen können, neue ästhetische oder ethische Leitbilder hervorbringen. ${ }^{33}$

9. Das Erzeugen von normativen Synästhesien kann beabsichtigt sein. Seit der Existenz großer sozialer Verbände, die auf das koordinierte Verhalten ihrer Mitglieder angewiesen, aber außer Stande sind, einen rationalen Herrschaftsdiskurs in Permanenz zu organisieren, hat das zielgerichtete Ausnutzen normativer Kategorienfehler sogar einen wesentlichen Anteil an der Stabilisierung gesellschaftlicher und politischer Autorität. Der Gebrauch dieses Instruments ist allerdings mit zahlreichen Risiken behaftet, da sich die Wirkungen eines assoziativen Mechanismus naturgemäß nur in begrenztem Umfang voraussehen und steuern lassen. Die Fehleranfälligkeit wird gemeinhin unterschätzt, ebenso wie die Bedeutung spontaner, nicht intendierter normativer Synästhesien (dazu III. s.v. Chocks). Wenn kategorial verschiedene Wertungen interagieren und sich zu neuartigen Aggregaten zusammenschließen, geschieht das unabhängig davon, ob die Verknüpfung beabsichtigt war - so wie die epikureischen Atome ohne Beteiligung eines göttlichen Choreographen, vielmehr allein aufgrund zufälliger Abweichungen kollidieren, sich verbinden und dadurch Neues schaffen.

\section{III.}

In dem sich anschließenden kleinen Glossar sind in alphabetischer Reihenfolge Grundbegriffe verzeichnet und erläutert, die einen Bezug zur normativen Synästhesie aufweisen. Dahinter steht die Beobachtung, dass das Phänomen in verschiedenen fachlichen Kontexten thematisiert wird - in der kognitiven Psychologie ebenso wie in der Soziologie, Ethnologie, Kunstgeschichte, Philosophie und in den Kultur-, Kommunikations- und Literaturwissenschaften. Da die Rubriken und Fachbegriffe stark voneinander abweichen und es bislang nur wenige Ansätze zu einer übergreifenden, vergleichenden Betrachtung gibt, mag es hilfreich sein, korrespondierende Konzepte kenntlich zu machen. Es versteht sich von selbst, dass in dem gegebenen Rahmen allenfalls kursorische Übersichten und Hinweise möglich sind.

\section{Analoges Denken/Metapher}

Wenngleich auch früheren Zeiten die menschliche Neigung zur »Bildlichkeit« selbstverständlich nicht entgangen war, so ist doch erst seit einigen Jahrzehnten unsere Befähigung, »sich mithilfe des Alten und Bekannten das Neue und Unbekannte zu erschließen «, ${ }^{34}$ Gegenstand systematischer wissenschaftlicher Untersuchungen. ${ }^{35}$ Analogien - als Oberbegriff für das habituelle Verknüpfen gedanklicher Inhalte zur Erweiterung und Übertragung von Erfahrungswissen - sind in unserem Denken allgegenwärtig. Sie sind das »Herz des Denkens «. ${ }^{36}$

Im Mittelpunkt des Interesses steht die Metapher. ${ }^{37}$ Als Gründungsmanifest der kognitiven Metaphertheorie gilt gemeinhin die von George Lakoff und Mark Johnson 1980 veröffentlichte Schrift "Metaphors We Live By ${ }^{38}{ }^{38}$ Die kognitive Linguistik in der Tradition Lakoffs und Johnsons betont die Rolle der Metapher als zentrale Steuerungsinstanz des menschlichen Denkens. Sie verweist darauf, dass es so gut wie keinen Bereich unserer Geistestätigkeit gibt, der nicht durch und durch metaphorisch strukturiert ist. Der kognitive Ansatz hat vor allem konzeptuelle Metaphern (oder metaphorische Konzepte) im Blick. ${ }^{39}$ "Konzept « be-
33 Beispielsweise fand der Energieerhaltungssatz oder - noch allgemeiner der energetische Monismus um 1900 mittelbar oder unmittelbar Eingang in die künstlerischen und rechtswissenschaftlichen Diskurse (freilich begünstigt durch die sinnliche Präsenz neuer Energie(übertragungs)formen im privaten und öffentlichen Raum), vgl. Damler (2016b) 152-191; Asendorf (1989).

34 Hofstadter/SAnder (2014) 17.
35 Zum Folgenden bereits DAMLeR (2016b) 38-50.

36 Hofstadter/SANder (2014) 35.

37 Der Begriff der Metapher, so wie er hier verwendet wird, ist bewusst weit gefasst. Für andere Zwecke mögen Binnendifferenzierungen sinnvoll sein, vorliegend würden sie nur den Blick auf das Wesentliche verstellen in diesem Sinne auch Demandt (1978) 3 f.: »DerTerminus >Metapher steht für Worte, die aus ihrem ge- wöhnlichen Verwendungszusammenhang herausgenommen und auf einen anderen Sachverhalt ... angewandt werden.« Zum ebenfalls recht weiten Metapherbegriff der Aristotelischen Poetik vgl. Höffe (2012) $10 \mathrm{f}$. 38 Dt.: LAKoff/Johnson (2008).

39 Zur »conceptual metaphor« und ihrer Abgrenzung von »metaphorical linguistic expressions « vgl. Kövecses (2010) 4-6. 
deutet in dem Zusammenhang, dass wir in der Alltags- wie in der Wissenschaftssprache abstrakten Phänomenen ein kohärentes metaphorisches Bedeutungssystem unbewusst unterlegen, dass wir also einzelne metaphorische Ausdrücke zu größeren Sinnaggregaten zusammenschließen, ohne darüber zu reflektieren.

Eine konzeptuelle Leitmetapher kann im Laufe der Zeit durch eine andere ersetzt werden. ${ }^{40}$ So hat die statistische Auswertung von Artikeln einer führenden psychologischen Fachzeitschrift - der Psychological Review - ergeben, dass während einer Zeitspanne von etwa neunzig Jahren (1894-1975) mindestens drei verschiedene Ursprungsdomänen zur Repräsentation mentaler Prozesse im Umlauf waren. ${ }^{41}$ In den frühen Jahrgängen um 1900 dominierten konzeptuelle Metaphern aus dem Bereich der belebten Welt (»through lying, mind grows wary«; »ideas struggle with one another«) und der räumlichen Ordnung (»anything hiding in the background is not mental activity«). Von der Jahrhundertmitte an entstammten die Metaphern dann hauptsächlich dem Wortschatz der expandierenden Elektro- und Computerwissenschaften. ${ }^{\mathbf{4 2}}$

An Kants programmatische Skizze in der »Kritik der Urteilskraft « knüpft Hans Blumenberg an, ${ }^{\mathbf{4 3}}$ der in seinen 1960 erschienenen »Paradigmen zu einer Metaphorologie« den Versuch unternimmt, "an die Substruktur des Denkens heranzukommen, an den Untergrund, die Nährlösung der systematischen Kristallisationen ${ }^{\mathbf{4 4}}{ }^{\mathbf{Z}} \mathrm{Zu}$ diesem Zweck untersucht er philosophische Leitmetaphern in historisch-philologischer Perspektive, beispielsweise die metaphorische Repräsentation der "Wahrheit" als Licht ${ }^{\mathbf{4 5}}$ und als »energetische Qualität«: als Macht der Wahrheit, als vis veri, als la force invincible de la vérité. ${ }^{\mathbf{4 6}}$ Solchen "absoluten « Metaphern, die "nicht in Begrifflichkeit aufgelöst«, sondern nur durch andere Metaphern ersetzt werden können, ${ }^{47}$ schreibt Blumenberg eine epochale Bedeutung zu: »Ihr Gehalt bestimmt als Anhalt von Orientierungen ein Verhalten, sie geben einer Welt Struktur, repräsentieren das nie erfahrbare, nie übersehbare Ganze der Realität. Dem historisch verstehenden Blick indizieren sie also die fundamentalen, tragenden Gewissheiten, Vermutungen, Wertungen, aus denen sich Haltungen, Erwartungen, Tätigkeiten und Untätigkeiten, Sehnsüchte und Enttäuschungen, Interessen und Gleichgültigkeiten einer Epoche regulierten. ${ }^{\mathbf{4 8}}$

Der Metaphertheorie der kognitiven Linguistik noch etwas näher als Hans Blumenberg steht Harald Weinrich. In einem bereits 1958 veröffentlichten Aufsatz (»Münze und Wort. Untersuchungen an einem Bildfeld «) nimmt er deren Kernthesen weitgehend vorweg. ${ }^{49}$ Für Weinrich ist die Einzelmetapher in der Regel Teil eines größeren "Bildfeldes«. ${ }^{50}$ Dadurch werden ganze »Sinnbezirke« miteinander verbunden. Unterscheidet die kognitive Linguistik zwischen »Ursprungs-« und "Zieldomäne«, differenziert Weinrich im Anschluss an Jost Trier zwischen dem »bildspendenden « und dem »bildempfangenden Feld «. In dem von ihm angeführten Beispiel bildet der Sinnbezirk »Sprache« das bildempfangende und der Sinnbezirk "Geld « das bildspendende Feld. ${ }^{51}$

Die Stärke von Weinrichs Ansatz liegt darin, dass er Metaphern als eine unser Denken gestaltende Macht begreift und nicht lediglich als einen Spiegel unserer intellektuellen Biographie. Für Weinrich gehört die »metaphorische Logik« zu den wirkungsmächtigsten Formen der kognitiven Manipulation: "So wird nun verständlich, dass unser Weltbild entscheidend von unseren Bildfeldern (mehr als von den Wortfeldern!) bestimmt ist ... Das Bildfeld Wortmünze hat die Bildstelle Wortreichtum (copia verborum). Da die Menschen nun im allgemeinen der Ansicht sind, dass der Reichtum etwas Gutes ist, musste sich daraus mit der immanenten Logik des Bildfeldes ergeben, dass eine Sprache um so vollkommener ist, je mehr Wörter sie aufnimmt - durch Prägung oder Entlehnung. Der Denkzwang, der von dieser metaphorischen Logik ausgeht, hat viele Jahrhunderte die europäischen Sprachen und Literaturen beherrscht. ${ }^{52}$

Im Unterschied $\mathrm{zu}$ dem traditionellen, rein instrumentellen Verständnis der Metapher heben

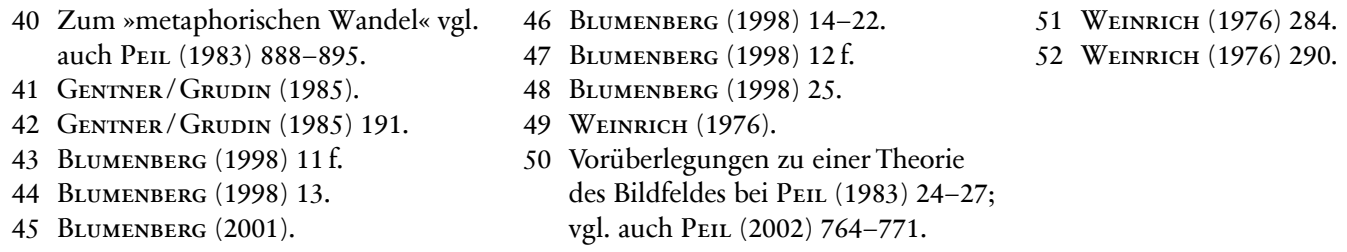

46 Blumenberg (1998) 14-22.

47 Blumenberg (1998) $12 \mathrm{f}$.

48 Blumenberg (1998) 25.

49 WeINRICH (1976).

50 Vorüberlegungen zu einer Theorie des Bildfeldes bei Peil (1983) 24-27; vgl. auch PeIL (2002) 764-771.

51 WEINRICH (1976) 284.

52 WeInRICH (1976) 290. 
Lakoff/Johnson, Blumberg und Weinrich den durch metaphorische Konzepte erzeugten »Denkzwang«, die ihnen innewohnende »Eigenlogik" hervor. Selbst wenn die Entscheidung für eine bestimmte Metapher bewusst erfolgt, gerät der Gebrauch leicht außer Kontrolle. Darüber hinaus gibt es Anhaltspunkte, dass schon die Analogiebildung als solche, die Auswahl der Metaphern eigenen - in funktionaler Hinsicht - »irrationalen« Gesetzen folgt. ${ }^{53}$ Maßgeblich ist oft die aktuelle Verfügbarkeit, die Präsenz eines Bildfeldes zum Zeitpunkt der Entscheidung und nicht eine wie auch immer geartete strukturelle Ähnlichkeit oder sachliche Richtigkeit. Maßgeblich können sein die Geräusche und Gerüche, denen wir ausgesetzt sind, die Lichtverhältnisse in den Räumen, in denen wir uns aufhalten, die Beschaffenheit Farbe, Form, Gewicht - der Gegenstände, die sich in unserer Umgebung befinden, und vieles mehr.

Damit ist die Brücke geschlagen zu normativen Kategorienfehlern, die so gesehen als ein Sonderfall metaphorischer Entgleisung gelten können. Eine Handlungsmaxime ist dann fehlerhaft, wenn sie auf der schieren Präsenz eines (inadäquaten) Leitbildes basiert und nicht einer sachlichen Notwendigkeit entspricht. Um es an einem Beispiel (rechts-)politisch-ästhetischer Grenzüberschreitung zu illustrieren: Nach traditioneller Auffassung und nach dem Selbstverständnis der involvierten Akteure ist prima causa der Forderung nach einer "gläsernen« oder »transparenten« Staatsgewalt das sachlich begründete Verlangen nach einem Mehr an Publizität und Öffentlichkeit. Wer hingegen das Eigengewicht und die Prägekraft ästhetischer Ideale wie »Durchsichtigkeit « und »Symmetrie» ernst nimmt, muss die Möglichkeit in Erwägung ziehen, dass es sich genau umgekehrt verhält, dass also solche Ideale in ihrer synästhetischen Mehrdeutigkeit das Verlangen nach uneingeschränktem Zugang zu staatli- chen Informationen überhaupt erst erzeugen oder jedenfalls überhöhen und verstärken.

\section{Attraktivitätsstereotyp}

Die bekannteste und am besten untersuchte Variante der Wertungskorrespondenz ist das Attraktivitäts- oder Schön-ist-gut-Stereotyp. Spätestens seit Anfang der 1970er Jahre ${ }^{54}$ gibt es wissenschaftlich belastbare Belege dafür, dass Schönheit das nach den gängigen Schönheitsidealen "gute» Aussehen eines Menschen - signifikant die Wahrscheinlichkeit erhöht, von seinen Mitmenschen als moralisch integer und intelligent qualifiziert $\mathrm{zu}$ werden, obwohl die eine Eigenschaft in keinem Zusammenhang mit der anderen steht.

Dieser Fehlschluss lässt sich nicht oder nur bis zu einem gewissen Grade mit gesellschaftlichen und kulturellen Prägungen erklären. Zum einen tritt der Effekt nachweislich bereits bei Kleinkindern auf, ${ }^{55}$ zum anderen operieren auch Angehörige anderer (nicht-westlicher) Kulturkreise mit der Schön-ist-gut-Hypothese. ${ }^{56}$ Für die Interferenzen sind möglicherweise Überschneidungen der involvierten neuronalen Areale (mit-)verantwortlich (»shared neural mechanisms of aesthetic and moral judgments«). ${ }^{57} \mathrm{Da}$ das Attraktivitätsstereotyp nicht nur die Bewertung schulischer Leistungen beeinflusst, sondern auch den Ausgang von Gerichts- und Verwaltungsverfahren, gibt es inzwischen Forderung nach einer Ausweitung der Antidiskriminierungsgesetzgebung auf Fälle ästhetisch bedingter Benachteiligung. ${ }^{58}$

\section{Chocks}

Je höher die Dichte der Reize und je kürzer die zeitlichen Abstände zwischen ihnen, desto größer ist die Wahrscheinlichkeit, dass Werturteile verkoppelt und auf unterschiedliche Sachverhalte
53 Einen aktuellen Überblick über den Stand der Forschung bietet LANDAu / Robinson/Meier (2014).

54 Dion/Berscheid/WALSTER (1972); zusammenfassend LANGLOIS / Kalakanis / Rubenstein / LaRson / Hallam / Smoot (2000) 404.

55 Ramsey/Langlois (2002).

56 Nur wird das "Gute« unter Umständen abweichend definiert: WHEELER/Kim (1997).
57 Tsukiura/Cabeza (2011); vgl. ferner (zu den gleichwohl vorhandenen Unterschieden) WANG / Mo/Mo/ TAN / CANT / ZHONG / CUPCHIK (2015). 58 Vgl. Rhode (2010). 
projiziert werden. ${ }^{59}$ Außerdem beeinträchtigt eine Reizüberflutung die Fähigkeit zum analytisch-kategorialen Denken. Die sich im 19. Jahrhundert stark beschleunigende Urbanisierung kommt daher als ein historischer Faktor in Betracht, der normative Kategorienfehler auf gesellschaftlicher Ebene begünstigt.

Schon Georg Christoph Lichtenberg, Heinrich Heine und Wilhelm von Humboldt waren gebannt von der Gleichzeitigkeit der Geschehnisse, wenn sie aus dem beschaulichen Deutschland nach London oder Paris reisten. ${ }^{60}$ Georg Simmel hat zu Beginn des 20. Jahrhunderts als Essentialia der Großstadterfahrung genannt »die rasche Zusammendrängung wechselnder Bilder, den schroffen Abstand innerhalb dessen, was man mit einem Blick umfasst, die Unerwartetheit sich aufdrängender Impressionen ${ }^{\mathbf{6 1}}{ }^{1}$ In ähnlicher Weise hat Walter Benjamin das Stadtleben als eine Abfolge »täglicher Chocks" und »Kollisionen« beschrieben. ${ }^{62}$ In den beiden Jahrhundertromanen, in James Joyces »Ulysses « und Alfred Döblins »Berlin Alexanderplatz«, geht es um nichts anderes als um das minuziöse Abbilden solcher »Kollisionen«, um das Kombinieren und Zusammendenken des Unzusammenhängenden in der Absicht, dadurch neue Bewusstseinsinhalte zu erzeugen. ${ }^{63}$

\section{Kalokagathie}

Der Begriff leitet sich vom altgriechischen kalós (»schön«) und agathós (»gut«) $\mathrm{ab}^{64}$ und bezeichnet im engeren Sinn ein Gesellschafts- und Charakterideal: die Vereinigung von physischer Schönheit und Adelstugenden. ${ }^{65}$ Derartige Verknüpfungen finden sich auch in anderen Kulturräumen und Sprachen. So besteht eine Verwandtschaft zwischen dem englischen fair/fairness ("gerecht/ anständig«) und dem althochdeutschen fagar (»schön/attraktiv«), eine Doppeldeutigkeit, die in der gehobenen Sprache nie ganz verloren gegangen ist (»a fair young maid «). ${ }^{66}$

Das zivilisatorische Leitbild hat in der griechischen Philosophie Spuren hinterlassen. ${ }^{67}$ Der Gedanke, dass die Attribute "schön «, "gut « und "gerecht" verschiedene Seiten ein und derselben Medaille darstellen, zieht sich wie ein roter Faden durch Platons Werk. Er begegnet uns im »Symposion« (201c; 204e), im »Gorgias" (476b; 476e) und »Protagoras« (358) ebenso wie in den staatstheoretischen Abhandlungen, in der "Politeia « und in den »Nomoi«. So heißt es in der zuletzt genannten Schrift (859d): »Über die Gerechtigkeit im allgemeinen und über die gerechten Menschen, Dinge und Handlungen sind wir uns doch alle irgendwie einig, dass dies alles schön ist; selbst wenn daher jemand behaupten wollte, dass die gerechten Menschen, auch wenn sie körperlich hässlich sind, dennoch aufgrund eben ihrer vollkommen gerechten Gesinnung vollkommen schön seien, so würde er mit einer solchen Behauptung wohl in keinem Fall etwas Verkehrtes zu sagen scheinen. ${ }^{68}$ In der Politeia (444e) setzt Platon »Tugend « mit »Gesundheit und Schönheit und Wohlbefinden der Seele" gleich sowie »Schlechtigkeit« mit »Krankheit und Hässlichkeit und Schwäche«, um daraus eine Handlungsempfehlung abzuleiten: »Führen nun nicht auch schöne Beschäftigungen zum Besitz der Tugend, hässliche aber zur Gerechtigkeit? « ${ }^{69}$

Hingegen ist es in Platons Deutung des "Sonnengleichnisses « das Gute, das Schönheit und Wahrheit hervorbringt (516b-c): »Was ich wenigstens sehe, das sehe ich so, dass zuletzt unter allem Erkennbaren und nur mit Mühe die Idee des Guten erblickt wird, wenn man sie aber erblickt hat, sie auch gleich dafür anerkannt wird, dass sie für alle die Ursache alles Richtigen und Schönen ist, im Sichtbaren das Licht und die Sonne, von der
59 Zum Folgenden bereits DamLer (2016a) $10 \mathrm{f}$.

60 Vgl. Möвius (2000) 43-45; STÜHLER (2002) $14 \mathrm{ff}$.

61 Simmel (2006) 9.

62 »Haptische Erfahrungen ... traten optischen an die Seite, wie der Inseratteil einer Zeitung sie mit sich bringt, aber auch der Verkehr in der großen Stadt. Durch ihn sich zu bewegen, bedingt für den einzelnen eine Folge von Chocks und von Kollisionen« - Benjamin (1974) 126.
63 Vgl. BecKer (1993) 288 f.; Möbius (2000) 429-457.

64 Zur Begriffsgeschichte PapILlon (1998).

65 Zur Kalokagathia als Adelsideal in der griechischen Antike MeIER (2000) 25-34. Kritisch zu dem gängigen Verständnis der Kalokagathia als Verknüpfung ästhetischer und moralischer Persönlichkeitselemente HORN (2005).

66 The Oxford English Dictionary (1989) 670-673, s.v. »fair«.
67 Zum Folgenden bereits DamLer (2016b) 193-202.

68 Platon (2011b) 195.

69 Platon (2011a) 361. 
dieses abhängt, erzeugend, im Erkennbaren aber sie allein als Herrscherin Wahrheit und Vernunft hervorbringend, und dass also diese sehen muss, wer vernünftig handeln will, es sei nun in eigenen oder in öffentlichen Angelegenheiten. ${ }^{\mathbf{7 0}}$

In Anbetracht der Wirkungsmacht der platonischen Lehren verwundert es nicht, dass die Losung kalòs kai agathós zu keiner Zeit in Vergessenheit geriet und als Leitmotiv in der europäischen Philosophie- und Geistesgeschichte nun schon seit mehr als zwei Jahrtausenden erklingt. Für die neuzeitliche Rezeptionsgeschichte war vor allem die Verbindung zwischen Rationalismus und Platonismus in der Philosophie der Cambridge Platonists, namentlich im Werk Shaftsburys, wichtig ${ }^{71}$ (moral sense ${ }^{72}$ ).

In Deutschland erreichte die intellektuelle Beschäftigung mit der Trias des »Wahren, Schönen, Guten ${ }^{73}$ und der Denkfigur der »schönen Seele ${ }^{74}$ im späten 18 . und frühen 19. Jahrhundert ihren Höhepunkt. Entschieden das Verbindende betonte beispielsweise Johann Gottfried Herder in seiner Spätschrift »Kalligone«. Erschienen im Jahre 1800, lässt sie sich als Programm und zugleich Genealogie alteuropäischer sittlich-ästhetischer Bildung lesen. Gleichgesinnte, wie er selbst »von der Fackel erleuchtet, in der das Wahre, Schöne und Gute, als ein dreifarbiger Stral erschien«, erblickte Herder in den Italienern Dante und Petrarca, in den Franzosen Crousaz, de Poully, Diderot, Rousseau und Montesquieu, in den Engländern Shakespeare, Milton, Pope, Young, Shaftesbury, Addison, Johnson, Cumberland, Hurd, Wharton und Webb, schließlich in seinen Landsleuten Lessing, Mendelssohn, Kästner, Baumgarten und Winckelmann. ${ }^{75}$ Andere haben diese Liste später noch ergänzt um die ritterliche Poesie (»bonté et beauté«), Thomas von Aquin (»pulchrum respicit vim cognoscitivam «), Leonardo da Vinci (»In der Wahrheit ist die Schönheit, in der Schönheit die Wahrheit«), Hume (»beauty of honesty«), Wilhelm von Humboldt (»ergreifende Schönheit der Tugend«) und Herbart (»Der sittliche Geschmack als Geschmack überhaupt ist nicht verschieden von dem poetischen, musikalischen, plastischen Geschmack «). ${ }^{76}$

Das Ideal des "Sittlichschönen« war in der Literatur und Philosophie um 1800 so präsent, dass niemand, nicht einmal Immanuel Kant, es mit einem einzigen Federstrich aus der Welt zu schaffen vermochte. In der Dichtung Schillers und Goethes existierte die Trias des »Wahren, Schönen, Guten« fort, etwa in Goethes Künstlerlied in den "Wanderjahren«, in dessen »Epilog zu Schillers Glocke $"{ }^{77}$ oder in Schillers Gedicht »Die Künstler» von 1789, das die Schönheit als die im Voraus geoffenbarte Vernunft, Tugend und Wahrheit verherrlicht (»Was erst, nachdem Jahrtausende verflossen/Die alternde Vernunft erfand/Lag im Symbol des Schönen und des Großen/Voraus geoffenbart dem kindischen Verstand «). ${ }^{\mathbf{7 8}}$

Im 19. Jahrhundert erlebte die Trias im Milieu der Künstler und Geisteswissenschaftler einen beispiellosen Niedergang. ${ }^{79}$ Scharf kritisierte Nietzsche die »Vermoralisierung der Künste« und fügte hinzu: "An einem Philosophen ist es eine Nichtswürdigkeit zu sagen: das Gute und das Schöne sind Eins: fügt er gar noch hinzu rauch das Wahre`, so soll man ihn prügeln. Die Wahrheit ist hässlich: wir haben die Kunst, damit wir nicht an der Wahrheit zu Grunde gehen. ${ }^{\mathbf{8 0}}$ Nietzsches Verdikt machten sich im 20. Jahrhundert so gegensätzliche Denker wie Max Weber, Heidegger und Adorno zu eigen. ${ }^{81}$ Aber das bedeutete noch lange nicht, dass der bildungsbürgerliche Mainstream, dem ein groBer Teil der Juristen und anderer Funktionseliten entstammte, mit der avantgardistischen Polemik gegen die Instrumentalisierung der Kunst einverstanden war (soweit er sie überhaupt zur Kenntnis nahm), ja es stellt sich die Frage, ob die Formel des »Wahren, Guten, Schönen« nach ihrer Desavouierung durch die Avantgarde nicht erst ihr goldenes Zeitalter erlebte, abzulesen unter anderem an den erbaulichen Sinnsprüchen und prunkenden Devisen, die seit dem späten 19. Jahrhundert die Dachfriese deutscher Opernhäusern zieren.

Ein Blick auf die Naturwissenschaften zeigt, dass selbst ihrer Prägung nach nüchterne Naturen
70 Platon (2011a) $564 \mathrm{f}$.

71 Kurz (2015) $23 \mathrm{f}$.

72 Zur (begrenzten) Moral-Sense-Rezeption in Deutschland vgl. ENGBERs (2001).

73 Kurz (2015).

74 WokaleK (2011).
75 Herder (1955) $65 \mathrm{f}$.

76 Triepel (1947) 26-30.

77 Kurz (2015) 79-88.

78 SCHILler (2005) 202.

79 Kurz (2015) 93-104.

80 NietzsChe (1980) 500.

81 Kurz (2015) 103. 
dazu neigten, eine Verbindung herzustellen zwischen Schönheit, Tugend und Wahrheit. Ausgerechnet Mathematiker, Quantenphysiker und Molekularbiologen haben sich im 20. Jahrhunderts als Herders gelehrigste Schüler erwiesen. So bekannte Heisenberg in seinem viel beachteten Vortrag "Die Bedeutung des Schönen in der exakten Naturwissenschaft « (1970), schon als Gymnasiast habe er mathematische Abhandlungen "ganz unmittelbar als schön « erlebt. ${ }^{82}$ Und Paul Dirac, ein weiterer Pionier der Quantenphysik, schrieb über Einstein: "When Einstein was working on building up his theory of gravitation he was not trying to account for some results of observations. Far from it ... His entire procedure was to search for a beautiful theory ... Somehow he got the idea of connecting gravitation with the curvature of space. He was able to develop a mathematical scheme incorporating this idea. He was guided only by consideration of the beauty of these equations. $"{ }^{83}$ Für Dirac selbst spielte die Aussicht auf ästhetischen Genuss ebenfalls eine große Rolle. Der experimentelle Nachweis sei zweitrangig: "It is more important to have beauty in one's equitation than to have them fit experiment. ${ }^{84}$

Die Liste derer, die ähnlich dachten und fühlten wie Heisenberg, Einstein oder Dirac lässt sich problemlos um weitere illustre Namen ergänzen. Ernest Rutherford verglich - wiederum mit Blick auf Einstein - eine naturwissenschaftliche Entdeckung mit einem Kunstwerk und bewertete sie folglich nach künstlerischen Qualitäten. ${ }^{85}$ Jules Henri Poincaré behauptete gar, ein Wissenschaftler studiere die Natur nicht, weil dies von Nutzen sei, sondern er tue es, weil es ihm Freude bereite, und es bereite ihm Freude, weil es schön sei. ${ }^{86}$ Auch Physiker der nachfolgenden Generation haben sich bemüht, ihr Wirken ästhetisch zu legitimieren, etwa Subrahmanyan Chandrasekhar in seinem Vortrag "Beauty and the Quest for Beauty in Science" (1979). Stets habe er versucht, zitiert er Hermann Weyl, das Wahre mit dem Schönen zu verbinden, aber wenn eine Entscheidung unvermeidlich gewesen sei, habe er ausnahmslos das Schöne gewählt. ${ }^{87}$
In den modernen Technik- und Naturwissenschaften funktionieren Ergebniskontrolle und Rückkoppelung im Normalfall zu gut, als dass die Schönheit-ist-Wahrheit-Formel eine nachhaltige Wirkung entfalten könnte. Für andere Lebensbereiche gilt das nicht in dem gleichen Maße. Insbesondere politische Prozesse sind weit anfälliger für ästhetische Interferenzen, da in Massenund Mediengesellschaften ein streng rationaler Diskurs nicht gewährleistet ist. In diesem Rahmen könnte die populäre Kalokagathie-Apotheose als ein variabler kultureller Faktor die gesellschaftliche Akzeptanz einer Reihe normativer Kategorienfehler im 19. und 20. Jahrhundert ${ }^{\mathbf{8 8}}$ befördert haben.

\section{Konsumismus/Sachkultur}

Mit dem Phänomen, die moralischen und intellektuellen Qualitäten einzelner Personen oder sozialer Gruppen allein anhand der von ihnen verwalteten und konsumierten Sachgüter zu beurteilen, beschäftigen sich seit dem späten 19. Jahrhundert die Sozialwissenschaften, Ethnologie, Kulturanthropologie und material culture studies. Eine einheitliche Begrifflichkeit und ein einheitlicher theoretischer Rahmen haben sich - soweit ersichtlich - bislang noch nicht herausgebildet, was die Rezeption und ideengeschichtliche Kontextualisierung erschwert.

Der beispiellose Aufstieg einer identitätsstiftenden Waren- und Sachkultur seit dem späten 19. Jahrhundert hat seine Ursache im Zerfall traditioneller Sozialstrukturen und der damit einhergehenden Individualisierung, »Anonymisierung«, »Vereinzelung«. An die Stelle unmittelbarer Kommunikation mit Verwandten und Bekannten trat eine indirekte mit Fremden. Der demonstrative Konsum von Waren (Thorstein Veblens »conspicuous consumption «) wurde zum zentralen Instrument gesellschaftlicher Distinktion und stellt in modernen urbanen Gesellschaften oft die einzige Möglichkeit dar, mit der anonymen Nachbarschaft zu kommunizieren. ${ }^{89}$ Bereits Veblen hat auf die unter solchen Bedingungen sich verstärkende Neigung zu Kategorienfehlern hingewiesen, etwa
82 Heisenberg (1985) 370.

83 Dirac (1980) 44.

84 Dirac (1963) 47.

85 McAllister (1996) 14

86 Poincaré (1920) 15.

87 Chandrasekhar (1987) 65.
88 DAmler (2012).

89 JäCKel (2006) 42. 
wenn er bemerkt, dass »die Merkmale der Kostspieligkeit ... allmählich zu Merkmalen der Schönheit « werden. ${ }^{90}$ Darüber hinaus prägt die DingWelt zunehmend auch das intime self-fashioning. ${ }^{91}$

Ökonomische und politische Krisen bewirken nicht notwendigerweise eine Schwächung des Konsumismus, wie Helen und Robert Lynd in ihren berühmten Middletown-Studien gezeigt haben, ${ }^{92}$ ja in Deutschland stand der Waren-Fetisch in den politisch besonders turbulenten Jahren nach 1914/18 und 1939/45 in höchster Blüte. ${ }^{93}$ Im Jahr 1915, mitten im Krieg, erschien das »Deutsche Warenbuch«, ein Katalog der »Dürerbund-Werkbund-Genossenschaft «, der als »bilderreiches Preisverzeichnis der brauchbarsten, gediegensten und schönsten Massenware der Gegenwart" Einfluss nehmen wollte auf das Verhalten und Empfinden des deutschen Konsumenten. ${ }^{\mathbf{9 4}}$ Joseph Popp, der Verfasser der »Einführung«, stellte klar, dass man eine sittliche, zivilisatorische Mission verfolge, deren Schicksal über Wohl und Wehe der »allgemeinen Kultur« entscheide: »Die gute Ware fördert ein Volk nicht nur wirtschaftlich, sondern auch sittlich und künstlerisch. Jeder Besitz wirkt auf die Familie, als die Zelle der Gesellschaft, Gutes und Böses schaffend. Wir erfahren aus den Dingen der häuslichen Umgebung und des alltäglichen Gebrauches fördernde und hemmende Eindrücke, niedere und hohe Stimmungen, Auffassungen und Gewohnheiten, die unbewusst unser Leben beeinflussen und prägen. « 95

Fortwährend ist im »Deutschen Warenbuch" die Rede von der "Wahrhaftigkeit« der "guten Massenware «, ${ }^{96}$ dem »Charakter, Geist und Stimmungswert guter Werkstoffe «, ${ }^{97}$ von der »bösen Tat « der »unsittlichen Ware «, ${ }^{\mathbf{8}}$ »nicht nur für den einzelnen verderblich, sondern fürs ganze Volk, ja die ganze Kulturwelt «. ${ }^{\mathbf{9 9}}$ Explizit spricht Popp von „Erlösung«, die dem Käufer zweckmäßig gestalteter, "guter« Produkte zuteilwerde: »Der Kulturmensch der Gegenwart ist in Sachen des Geschmacks am wenigsten kultiviert, häufig von einer Überfülle des schlimmsten Schundes umgeben. Aus diesem Elend bringt nur eines Erlösung: das Schlechte durch das Gute zu ersetzen und hierfür unablässig zu werben. « ${ }^{\mathbf{1 0 0}}$

Ein Blick in Warenhauskataloge, Hausfrauenratgeber, Möbelprospekte und Architekturkompendien der 20er und 30er Jahre zeigt, dass das Plädoyer für eine »Erlösung durch Design" seit 1915 nichts an Aktualität eingebüßt hatte. Stilfibeln, die den säkularen Glauben an die Heilswirkung der "guten Ware" und der "guten Form" beschworen, überfluteten das Land. Die Designtheologie der Moderne war gleichsam das Surrogat für einen übergreifenden weltanschaulichen Konsens, den die maßgeblichen gesellschaftlichen Kräfte nach der Zäsur von 1914 nicht mehr herzustellen vermochten. An den Glaubenssätzen des modernen Formen- und Materialkatechismus kam niemand vorbei, sofern er nur in irgendeiner Weise am gesellschaftlichen Leben teilnahm. ${ }^{101}$

Die moralisierende Waren- und Formkritik überstand auch die nationalsozialistische Diktatur weitgehend unbeschadet. Paul Betts hat unlängst überzeugend nachgewiesen, dass sogar gerade im Dritten Reich und in der frühen Bundesrepublik das Industriedesign eine eminent wichtige Rolle bei der Identitätsfindung spielte: »There was no clear divide between 1932 and 1933 in German industrial aesthetics. Nazism provided little design innovation, nor did it ever really break from Weimar modernism. What was new was that industrial design enjoyed tremendous state backing to meet a variety of both economic and cultural ends. And even if the racist rhetoric of German design disappeared with the defeat of the Third Reich in 1945, the soaring idealism of design survived, the ideology of the design object as a marker of hope, loyalty, and transcendence continued unabated after the war. ${ }^{\mathbf{1 0 2}}$

Die Design-Kalokagathie der populären Warenkunde war eine wichtige Voraussetzung für die Irritationen der moderne Rechts- und Staatslehre
90 Veblen (2007) 132.

91 Vgl. Miller (2010a); Miller (2010b); Böнme (2016). Zur Präsenz »lebendiger Dinge« im (Text-)Kanon der Moderne Кіммісн (2011); ferner die Beiträge in Daston (2004). Zum "Ding-Fetisch « aus Sicht der Ethnologie und Religionsgeschichte КонL (2003).
92 Lynd/Lynd (1937) 242-294, 489; vgl. auch BrinkLEY (1999) 9-11.

93 Zum Folgenden bereits DamLer (2012) 15-19.

94 Popp (1915) XVII. Zum Kontext KöNIG (2009) 63-70, 82-91.

95 Popp (1915) XVII.

96 Popp (1915) XVIII, XXXI.

97 Popp (1915) XVIII.

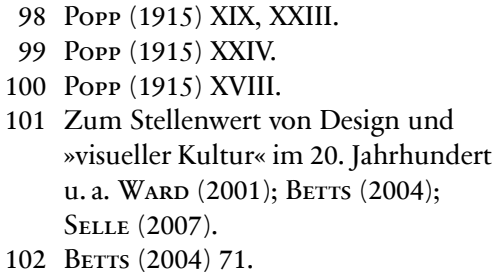

98 Popp (1915) XIX, XXIII

100 Popp (1915) XVIII. „visueller Kultur« im 20. Jahrhunder u. a. WARD (2001); BetTS (2004);

102 Betts (2004) 71. 
durch die Gestaltungs- und Architekturdiskurse des 20. Jahrhunderts. Denn wer von Gebäuden und Gebrauchsgegenständen Heil und Erlösung erwartet, dem fällt es nicht allzu schwer, in einem zweiten Schritt aus Formen und Materialen rechtsund staatstheoretische Prinzipien »herauszulesen«.

\section{Montage der Attraktionen}

Der Film mit seinen kurzen Bildsequenzen eignet sich besonders, um normative Kategorienfehler zu erzeugen. ${ }^{\mathbf{1 0 3}}$ Blieb die literarische Montage letztlich immer eine Technik der Avantgarde, so hat die filmische Montage ein sehr viel größeres Publikum erreicht und ist von allen politischen Lagern vereinnahmt worden. Meisterhaft beherrschte Sergej Eisenstein dieses Metier, der mit seiner "Montage der Attraktionen« die Filmkunst revolutionierte und nicht nur diese, sondern überhaupt den Umgang mit Bildern im öffentlichen Raum zum Zweck der politischen Einflussnahme. ${ }^{104}$

Der Kunstgriff bestand darin, Bilder, die an sich in keiner logisch-kausalen Beziehung zueinander stehen, so zu montieren, dass sie einen Sinn ergeben und den Zuschauer zur Parteinahme veranlassen, wie in einer berühmten Sequenz im Film "Streik" von $1925 \mathrm{zu}$ besichtigen, die deshalb so berührt, weil Eisenstein in rascher Abfolge abstoßende Szenen aus einem Schlachthof mit Bildern von der Ermordung streikender Arbeiter kombiniert. Indem Eisenstein ferner die Hauptakteure des Films für einen kurzen Moment in Tiergestalten verwandelt, visualisiert er deren Charaktereigenschaft so prägnant, dass eine Umdeutung und Rehabilitierung nicht mehr möglich ist.

»Dieses Herangehen", schreibt Eisenstein, »verändert auf radikale Weise die Möglichkeiten in den Konstruktionsprinzipien einer seinwirkenden Struktur (eine Aufführung im Ganzen): anstelle der statischen >Widerspiegelung < eines vom Thema her erforderlichen Ereignisses und der Möglichkeit, es ausschließlich mit Hilfe von Wirkungen zu gestalten, die logisch mit der Handlung verknüpft sind, wird eine neue Methode vorgeschlagen - die freie Montage von willkürlich ausgewählten, selbständigen (auch außerhalb dieser vorgegebenen Komposition und Handlungslinie funktionierenden) Einwirkungen (Attraktionen), allerdings mit einer genauen Orientierung auf einen bestimmten thematischen Endeffekt - die Montage der Attraktionen. ${ }^{105}$

Dass Eisenstein die Inspiration für seine Montagetechnik von dem Erleben der Großstadt empfing (s.v. Chock), bekannte er beiläufig in einer autobiographischen Notiz, in der er den Begriff »Montage" verteidigte, der, wie er betonte, halb in der Sphäre des Produktionsbetriebes, halb in der Sphäre der music hall zu Hause sei. »Beide sind sie Produkte des Urbanismus, wie wir alle in jenen Jahren furchtbar urbanistisch waren. ${ }^{106}$

\section{Norm/normatives Implantat}

Der Zusatz »normativ« bringt zum Ausdruck, dass Gegenstand der normativen Synästhesie Werturteile, "Werte und Bewertungen ${ }^{\mathbf{1 0 7}}$ jeder Art, sind. Fragen der Rechtfertigung, der Normenbegründung, spielen keine Rolle. ${ }^{108}$ Die habituelle Verknüpfung bewirkt oder erleichtert einen Austausch von Begriffen und Denkfiguren zwischen verschiedenen Wertekategorien. "Normativ« bedeutet im vorliegenden Zusammenhang demnach nicht, dass ausschließlich oder auch nur in erster Linie normative, vorschreibende statt beschreibende (deskriptive) Sätze verschaltet werden.

Dem Prozess der Naturalisation, der Übersetzung (im übertragenden Sinn) kommt aufgrund des großen Abstandes zwischen den Normenkategorien eine herausragende Bedeutung zu. ${ }^{109}$ Zwar bedürfen auch rechtliche "Transplantate «10 einer Anpassung an die neuen Verhältnisse (schon die
103 Zum Folgenden bereits DamLer (2016a) $13 \mathrm{f}$.

104 Vgl. nur Möвıus (2000) insbes. 359-368.

105 Eisenstein (2006) 12.

106 EIsenstein (1974) $193 \mathrm{f}$.

107 Möllers (2015) 18. Zum Norm-Begriff aus philosophischer und sozialwissenschaftlicher Sicht JAEGGI (2014) 144-148; AHrens u. a. (2011); Stemmer (2008).
108 Vgl. auch Möllers (2015) 19.

109 Allgemein zur Öffnung gegenüber anderen Formen und Vorstellungen von Normativität als Forschungsperspektive Duve (2014). Von einem bemerkenswerten Gespür für Übergangsformen zwischen weit entfernten Normenkategorien - namentlich zwischen juristischen und ästhetischen Normen - zeugt die auf Christian Thomasius zurückgehende Lehre vom »ius decori« - dazu eingehend Vec (2010); Vec (2009) 160. Zum normativen Anspruch ästhetischer Urteile im Kontext der modernen Kunsttheorie MöLlers (2015) 260-265.

110 Vgl. Watson (1974). 
Übersetzung eines Gesetzes oder einer rechtswissenschaftlichen Abhandlung in eine andere Sprache beinhaltet eine beachtliche Akkulturationsleistung ${ }^{111}$ ), doch es ist immerhin - um im Bild zu bleiben - organisches Material, das Gewebe eines Menschen oder (bei xenogener Transplantation) zumindest eines Lebewesens, das verpflanzt wird. Normative "Implantate " hingegen sind in jeder Hinsicht Fremdköper, künstliche, unorganische Substanzen, die erst nach und nach, im Verlaufe eines langwierigen Fabrikationsverfahrens eine Form und Beschaffenheit erhalten, die es ermöglicht, Wirkungen im neuen Umfeld zu erzeugen. Dieser Prozess - und nicht bereits der Kategorienwechsel als solcher - kann (und wird in der Regel) kategorienadäquate normative Sätze hervorbringen, Sätze, die nicht beanspruchen, zur Welt zu passen, sondern darauf zielen, dass die Welt zu ihnen passt (normativer »direction of fit ${ }^{\mathbf{1 1 2}}$ ).

\section{Raum-, Hygiene-, Klang-, Proportions-»Werte»}

Seit einigen Jahren beschäftigt sich die experimentelle Psychologie mit der Verwechslung normativer Kategorien, wie sie in der Alltagssprache zu Tage tritt (s.v. »Kalokagathie«). ${ }^{\mathbf{1 1 3}}$ Die Diskussion ist im Fluss und alles andere als abgeschlossen. Über Sinn und Unsinn einiger Experimente gibt es eine lebhafte Auseinandersetzung. ${ }^{114}$ Auch ist nicht immer erkennbar, in welchem Verhältnis die experimentell gewonnenen Ergebnisse zueinander stehen. Dennoch, trotz so mancher Unstimmigkeiten, weisen die Resultate eine gewisse Kohärenz auf, die in begrenztem Umfang Rückschlüsse und Verallgemeinerungen zulassen.

Große Beachtung gefunden haben Studien, die belegen, dass Empfindungen wie Ekel unser moralisches Urteil beeinflussen können. ${ }^{\mathbf{1 1 5}}$ So hat man die Teilnehmer eines Experiments just in dem Moment unangenehmen Gerüchen ausgesetzt, in dem sie Urteile zu ethisch brisanten Konfliktlagen abgeben mussten. Der Versuch wurde mit anderen Probanden wiederholt, diesmal aber ohne vorher die Örtlichkeit olfaktorisch zu kontaminieren. Ein Abgleich der Antworten brachte ans Licht, ${ }^{\mathbf{1 1 6}}$ dass diejenigen Versuchsteilnehmer, die mit einer Geruchsbelästigung konfrontiert waren, das zu bewertende Verhalten kritischer beurteilten als diejenigen, die unter neutralen Bedingungen den Fragebogen ausfüllten. ${ }^{\mathbf{1 1 7}}$

Wenn das Empfinden von Ekel zu einer strengeren moralischen Bewertung führt, liegt die Vermutung nicht fern, dass die Konfrontation mit Konzepten wie »Sauberkeit« oder »Reinheit« genau das Gegenteil bewirkt. Auch dazu gibt es Untersuchungen: Wiederum mussten die Versuchsteilnehmer eine Anzahl von ethisch problematischen Handlungen beurteilen. Diejenigen, die unmittelbar vorher einen mit Vokabeln wie "gewaschen«, »rein « und »sauber« angereicherten Text gelesen hatten, gaben im Durchschnitt eine mildere Bewertung ab als jene, deren Lektüre diese Begriffe nicht enthielt. ${ }^{118}$ Die gleiche Differenzierung zeigte sich bei einer realen körperlichen Reinigung, als man nämlich einen Teil der Probanden aufforderte, sich vor dem Test die Hände zu
111 Dazu Duve (2016) 16 f. Vgl. auch Vogenauer (2015).

112 JaegGi (2014) $145 \mathrm{f}$.

113 Zum Folgenden bereits DamLer (2016b) 202-209.

114 Unstimmigkeiten belegt eine neuere Untersuchung zu hundert im Jahr 2008 in den Zeitschriften Psychological Science, Journal of Personality and Social Psychology und Journal of Experimental Psychology: Learning, Memory, and Cognition erschienenen Studien: Open Science Collaboration (2015). Die »Erfolgsquote« war bei kognitionspsychologischen Experimenten höher als bei sozialpsychologischen. Die Gründe für die Abweichungen sind, wie auch die Verfasser der Kontrollstudie betonen, unklar. Kritisch zur Belastbarkeit vieler »Wiederholungsstudien « (u. a. wegen geänderter Rahmenbedingungen): Schwarz/Strack (2014).

115 Schnall/Haidt/Clore/Jordan (2008) 1097-1099. Kritisch dazu LANDY / GoodwIN (2015); dagegen wiederum Schnall / Haidt / Clore/ Jordan (2015).

116 Schnall/Haidt/Clore/Jordan (2008) 1097-1099.

117 Die Unterschiede waren auch in den Folgeexperimenten nachweisbar. Diese variierten die Art der Reize: In einem Fall mussten die Probanden ihre Fragen in einem schmutzigen, unhygienischen Umfeld beantworten, in einem anderen Versuch hatten sie sich unmittelbar vor ihrer Befragung an einen Ekel erregenden Vorgang aus ihrem Leben zu erinnern, im vierten Experiment schließlich wurden ihnen Filmausschnitte mit unappetitlichen Szenen vorgeführt. Die Auswertung ergab, dass ein Tei der Probanden von vornherein völlig immun gegenüber den sinnlichen Manipulationen ist, ferner dass das Wissen der Teilnehmer um die Bedeutung körperlicher Empfindungen für sittliche Urteile den Manipulationseffekt reduziert und dass - drittens - der Manipulationseffekt in der Form wirklich nur bei sittlichen Urteilen auftritt und beispielsweise nicht bei der Bewertung politischer Sachverhalte (ohne ethische Implikationen) - Schnall / Haidt / Clore / JORDAN (2008).

118 Schnall / Benton/Harvey (2008) $1219 \mathrm{f}$. 
waschen, während andere Probanden diese Anweisung nicht erhielten. ${ }^{119}$ Allerdings ist sehr umstritten, wie gravierend die Effekte sind, die sich bei einer Manipulation mit Attributen der (Un)reinheit einstellen. ${ }^{\mathbf{1 2 0}}$ Immerhin bestreiten auch die meisten Kritiker der erwähnten Studien nicht, dass sich Interferenzen nachweisen lassen.

Wenn demnach das Vertauschen von sinnlichen und sittlichen Kategorien mehr ist als nur ein belangloses Spiel mit Worten, dann stellt sich die Frage, ob das Gleiche für das Verhältnis von »Wahrheit« und "Schönheit" gilt. So wie ästhetische Empfindungen Einfluss nehmen auf das sittliche Urteilsvermögen, könnten sie auch Interferenzen bei der Wahrheitsfindung erzeugen. Auch der Weiseste von uns, schreibt Nietzsche, werde "gelegentlich zum Narren des Rhythmus, sei es auch nur darin, dass er einen Gedanken als wahrer empfindet, wenn er eine metrische Form hat und mit einem göttlichen Hopsasa daher kommt. Ist es nicht eine sehr lustige Sache, dass immer noch die ernstesten Philosophen, so streng sie es sonst mit aller Gewissheit nehmen, sich auf Dichtersprüche berufen, um ihren Gedanken Kraft und Glaubwürdigkeit zu geben?« ${ }^{\mathbf{1 2 1}} \mathrm{Um}$ zu überprüfen, ob der Reim als solcher tatsächlich, wie Nietzsche behauptet, einem Gedanken »Kraft und Glaubwürdigkeit« verleiht, hat man Probanden gebeten, anzugeben, inwieweit die zur Begutachtung vorgelegten Aphorismen das menschliche Verhalten zutreffend beschreiben. Ihrem Inhalt nach waren die Aphorismen identisch, doch erhielt nur ein Teil der Probanden den Aphorismus in Form eines Reimes (z. B. »What sobriety conceals, alcohol reveals«). Diese Gruppe bewertet den Wahrheitsgehalt der Aussage höher als diejenigen Versuchsteilnehmer, die eine nicht gereimte Version zu bewerten hatte (»What sobriety conceals, alcohol unmasks«). ${ }^{\mathbf{1 2 2}}$ Nur wenn man alle Teilnehmer vorher explizit davor warnte, sich von der metrischen Form ablenken zu lassen, waren die Ergebnisse in beiden Versuchsreihen in etwa gleich. ${ }^{\mathbf{1 2 3}}$

Denkbar erscheint es auch, dass der Anblick symmetrischer Muster - ähnlich wie die Perzeption eines Reimes - das Urteil über den Wahrheitsgehalt einer Aussage zu beeinflussen vermag, da Symmetrien in nahezu allen Kulturen als angenehm, als »schön « empfunden werden. Also hat man Versuchsteilnehmern Punktmuster in Form einer Gleichung vorgelegt, die sie als wahr oder falsch bewerten sollten, wobei einige Muster symmetrisch, andere hingegen asymmetrisch angeordnet waren. Die Probanden hatten weniger als zwei Sekunden Zeit, um ihre Entscheidung zu treffen, eine Zeitspanne, die nicht ausreichte, um die Punkte korrekt abzuzählen. ${ }^{\mathbf{1 2 4}}$ Es stellte sich heraus, dass die Versuchspersonen eher Gleichungen mit symmetrischen als mit asymmetrischen Punktmustern für richtig hielten. ${ }^{\mathbf{1 2 5}}$

Das Phänomen lässt sich möglicherweise mit identischen Verarbeitungssystemen erklären: "Judgments of beauty and intuitive judgments of truth may share a common underlying mechanism. Although human reason conceptually separates beauty and truth, the very same experience of processing fluency may serve as a nonanalytic basis for both judgments. ${ }^{\mathbf{1 2 6}}$ Die Schnelligkeit oder Fluidität der Informationsverarbeitung indiziert, ob wir eine Aussage für »wahr,${ }^{\mathbf{1 2 7}}$ aber auch ob wir ein Objekt als »schön« empfinden. ${ }^{\mathbf{1 2 8}}$

Auf einen ähnlichen Mechanismus könnte es zurückzuführen sein, dass wir positive Begriffe (»Held«) schneller erkennen, wenn sie im oberen Teil eines Bildschirms auftauchen, und negative (»Lügner«) schneller, wenn sie im unteren Feld erscheinen. ${ }^{129}$ Aber nicht nur oben und unten sind mit moralischen Wertungen verknüpt, sondern auch rechts und links: Rechtshänder assoziieren
119 Schnall / Benton / Harvey (2008) 1220-1222. Vgl. ferner ZHONG/ STREJCEK / Sivanathan (2010) $860 \mathrm{f}$; LEE/SCHWARZ (2011).

120 LANDY / GoODWIN (2015); JoHnson / Wortman / Cheung / HeIn / Lucas / DONNELlan / Ebersole/ NarR (2016). Zur Bedeutung individueller Sensibilität vgl. Ong / MulletteGillman/KwOK/Lim (2014).

121 Nietzsche (1988) 442.

122 McGlone/ Tofighbakhsh (2000) $425 \mathrm{f}$.
123 McGlone / TOFighbaKhsh (2000) $425 \mathrm{f}$.

124 ReBer/Brun / Mittendorfer (2008) $1175 \mathrm{f}$.

125 ReBER/BRUn / MitTENDORFER (2008) 1176.

126 REBER/SCHWARZ/WINKIELMAN (2004) 377. Ebenso Reber/Brun/ Mittendorfer (2008) $1177 \mathrm{f}$.

127 Reber/UnKelbach (2010).

128 REBER/SCHWARZ/WINKIELMAN (2004).

129 Meier/Robinson (2004). 
den Raum zu ihrer Rechten mit positiven Ideen und Werten, den Raum zu ihrer Linken hingegen mit negativen. ${ }^{\mathbf{1 3 0}}$ Das könnte man vielleicht noch mit kulturellen und sprachlichen Prägungen erklären, da das Englische good mit right gleichsetzt, doch zeigte sich bei (ebenfalls englischsprachigen) Linkshändern der genau gegenteilige Effekt (good / left) und im Übrigen auch bei Rechtshändern, deren rechte Hand aufgrund eines Schlaganfalls gelähmt ist. ${ }^{131}$

\section{Synästhesie / cross-modale Korrespondenz}

Synästhesie bezeichnet das Zugleich-Wahrnehmen verschiedener Sinnesempfindungen. Der Begriff wird vorliegend schon deshalb im übertragenden Sinn gebraucht, weil es sich bei dem Verkoppeln von Wahrnehmungen um eine Anomalie, eine seltene Abweichung handelt, die bei maximal vier bis fünf Prozent der Bevölkerung auftritt. ${ }^{132}$ Die »normative Synästhesie« hingegen ist die Regel und nicht die Ausnahme. Einen Sonderfall stellt die Ideasthesie dar, das Verkoppeln von semantischen Repräsentationen und sensorischer Empfindung. ${ }^{133}$ Ein Fall von Ideasthesie liegt zum Beispiel vor, wenn bei der Lektüre eines bestimmten Buchstabens eine bestimmte Farbe aufgerufen wird. Hier kann von einem Zugleich-Wahrnehmen von Sinnesempfindung im eigentlichen Sinn (Ton Farbe, Geschmack - Form usw.) keine Rede sein, vielmehr löst eine semantische Repräsentation oder ein abstraktes Konzept ein sinnliches Begleitempfinden aus.

Von Synästhesien unterscheiden sich cross-modale Korrespondenzen dadurch, dass die Verknüpfung von Eigenschaften oder Aspekten unterschiedlicher Modalitäten nur latent vorhanden ist und sich nicht im bewussten Erleben der Eigenschaften manifestiert. Bereits 1929 gelang Wolfgang Köhler, einem der Pioniere der Gestaltpsychologie, der Nachweis, dass eine Affinität zwischen bestimmten Worten oder Klängen und be- stimmten Formen besteht: Die meisten der von ihm befragten Personen ordneten dem Wort »Maluma« runde und dem Wort »Takete« eckige Formen zu. Eine Wiederholung des Experiments zu Beginn des 21. Jahrhunderts - diesmal fanden die Phantasieworte »bouba « und »kiki« Verwendung bestätigten den Befund. Über 95\% der Versuchsteilnehmer gaben an, geschwungene Formen mit »bouba« und zackige mit »kiki« zu assoziieren. ${ }^{134}$ Weitere Beispiele für cross-modale Korrespondenzen sind die Paarungen Tonhöhe - räumliche Höhe, Tonhöhe - Helligkeit (hoch / hell, tief / dunkel) und Tonhöhe - Geschmack (hoch/süß, tief/ bitter). ${ }^{135}$ Cross-modale Korrespondenzen sind deshalb besonders interessant und relevant, weil sie bei allen Menschen und nicht nur bei einer kleinen Personengruppe auftreten.

Im Einzelnen ist allerdings noch vieles ungeklärt. So gehen die Meinungen darüber auseinander, ob cross-modale Korrespondenzen lediglich einen Sonderfall, eine »schwache« Spielart der Synästhesie oder ein aliud darstellen, ${ }^{\mathbf{1 3 6}} \mathrm{ob}$ man innerhalb der cross-modalen Korrespondenzen noch einmal zwischen Untergruppen differenzieren muss, die auf unterschiedlichen Wirkungsmechanismen basieren, ob die Verknüpfungen automatisch generiert werden, ${ }^{\mathbf{1 3 7}}$ ob cross-modale Korrespondenzen durch kulturelle Determinanten beeinflusst sind, ${ }^{\mathbf{1 3 8}}$ ob sie statistischen Häufigkeiten in der natürlichen Umgebung des Menschen Rechnung tragen (Beispiel: die Verknüpfung von Tonhöhe und räumlicher Ausdehnung als Reaktion auf die Resonanzeigenschaften von Körpern) usw. ${ }^{139}$

Erst recht muss offen bleiben, ob irgendein $\mathrm{Zu}$ sammenhang besteht zwischen wahrnehmungsbezogenen cross-modalen Korrespondenzen und ethisch-ästhetischen-epistemischen Wertungskorrespondenzen. Immerhin weisen beide Erscheinungen gewisse Übereinstimmungen auf. So gehören Helligkeit und süßer Geschmack genauso zwei grundverschiedenen, analytisch klar zu tren-
130 Casasanto (2009).

131 Casasanto/Chrysikou (2011).

132 Simner/Mulvenna/Sagiv/TsakaniSAKANIKOS / WITHERBY / FraSER / SCOTT/WARD (2006) 1028.

133 Vgl. JürgENS / NiCOLIĆ (2012); Mroczko-Wąsowicz/ Nikolić (2014).

134 Ramachandran/Hubbard (2001) 19.
135 Spence / Deroy (2013a) 246 (m. w. N.).

136 Spence / Deroy (2013b); Martino/ Marks (2001).

137 Spence/ Deroy (2013a).

138 Levitan et al. (2014).

139 Vgl. den Überblick bei PARISE (2015); SPENCE (2011). 
nenden Kategorien an wie Wahrheit und Schönheit - und trotzdem fällt es uns schwer, die eine Modalität ohne die andere zu denken.

Mit Blick auf die nicht minder grundverschiedenen Kategorien Farbe und Form - vielleicht schlechthin das Urbild einer kategorialen Differenz - hat Wassily Kandinsky, der von 1922 bis 1925 die Werkstatt für Wandmalerei am Bauhaus leitete, in Lehre und Forschung immer wieder auf psychologische Wechselspiele zwischen dem scheinbar Fernliegenden hingewiesen. Farbenlehre war für ihn ohne Formenlehre nicht denkbar. Kandinskys Korrespondenztheorie, die unter anderem auf der statistischen Auswertung seiner legendären, an alle Mitglieder des Bauhauses verteilten Fragebögen basierte (Rot, Gelb und Blau waren jeweils einem Dreieck, einem Quadrat oder einem Kreis zuzu- ordnen), besagt, dass Formen und Farben sich nicht indifferent zueinander verhalten, vielmehr der Kreis zur Farbe Blau, das Quadrat zur Farbe Rot und das Dreieck zur Farbe Gelb passe. ${ }^{\mathbf{1 4 0}}$

Die von Kandinsky propagierte »Kraft der Farbe ..., durch welche die Farbe eine gegebene Form verändern kann, so dass aus der gegebenen Form eine andere entsteht", wurde gleichsam zu einer der Geschäftsgrundlagen der abstrakten Kunst (»eine der wichtigsten Angelegenheiten der Bauhausaufgaben $)^{\mathbf{1 4 1}}$ und überdies des modernen Graphikdesigns, denn auch das - damals neuartige »Leitsystem «, das Herbert Bayer für das kleine Treppenhaus im Bauhaus-Hauptgebäude entwarf, nahm Bezug auf Kandinskys Korrespondenztheorie. $^{142}$

Bibliographie

- Ahrens, Johannes, Raphael Beer, Uwe H. Bittlingmayer, Jürgen Gerdes (Hg.) (2011), Normativität. Über die Hintergründe sozialwissenschaftlicher Theoriebildung, Wiesbaden

- Asendorf, Christoph (1989), Ströme und Strahlen. Das langsame Verschwinden der Materie um 1900, Gießen

- Becker, Sabina (1993), Urbanität und Moderne. Studien zur Großstadtwahrnehmung in der deutschen Literatur 1900-1930, St. Ingbert

- Benjamin, Walter (1974), Charles Baudelaire. Ein Lyriker im Zeitalter des Hochkapitalismus, Frankfurt am Main

- Betts, Paul (2004), The Authority of Everyday Objects. A Cultural History of West German Industrial Design, Berkeley u. a.

- Blumenberg, Hans (1998), Paradigmen zu einer Metaphorologie, Frankfurt am Main

- Blumenberg, Hans (2001), Licht als Metapher der Wahrheit. Im Vorfeld der philosophischen Begriffsbildung, in: Ders., Ästhetische und metaphorologische Schriften, hg. von Anselm Haverkamp, Frankfurt am Main, 139-171

- Böhme, Gernot (2016), Ästhetischer Kapitalismus, Berlin

- Brinkley, Alan (1999), Culture and Politics in the Great Depression, Waco

- Casasanto, Daniel (2009), Embodiment of Abstract Concepts. Good and Bad in Right- and Left-Handers, in: Journal of Experimental Psychology 138, 351-367

- Casasanto, Daniel, Evangelia G. Chrysikou (2011), When Left is »Right«. Motor Fluency Shapes Abstract Concepts, in: Psychological Science 22, 419-422

- Chandrasekhar, Subrahmanyan (1987), Beauty and the Quest for Beauty in Science, in: Ders., Truth and Beauty. Aesthetics and Motivation in Science, Chicago, London, 59-73

- Damler, Daniel (2012), Der Staat der Klassischen Moderne, Berlin

- Damler, Daniel (2016a), Konzern und Moderne. Die verbundene juristische Person in der visuellen Kultur 1880-1980, Frankfurt am Main

- Damler, Daniel (2016b), Rechtsästhetik. Sinnliche Analogien im juristischen Denken, Berlin

- Daston, Lorraine (Hg.) (2004), Things that talk. Object lessons from art and science, New York

- Demandt, Alexander (1978), Metaphern für Geschichte. Sprachbilder und Gleichnisse im historisch-politischen Denken, München

- Dion, Karen, Ellen Berscheid, Elaine Walster (1972), What is Beautiful is Good, in: Journal of Personality and Social Psychology 24, 285-290

- Dirac, Paul A. M. (1963), The Evolution of the Physicist's Picture of Nature, in: Scientific American 208, 45-53

- Dirac, Paul A. M. (2004), The Excellence of Einstein's Theory of Gravitation, in: Goldsmith, Maurice, Alan Mackay, James Wouldhuysen (Hg.) (1980), Einstein. The First Hundred Years, Oxford, 41-46

- Dörflinger, Bernd, Günter Kruck (Hg.) (2011), Über den Nutzen von Illusionen. Die regulativen Ideen in Kants theoretischer Philosophie, Hildesheim

140 ThüMmLer (1999) 457.

141 Zitiert nach ThüMmLER (1999) 458.

142 ThüMmLeR (1999) 458. 
- Duve, Thомas (2014), Rechtsgeschichte. Traditionen und Perspektiven, in: Kritische Vierteljahresschrift für Gesetzgebung und Rechtswissenschaft 97, 96-132

- Duve, Thomas (2016), Global Legal History - A Methodological Approach, Max Planck Institute for European Legal History Research Paper Series No. 2016-04, https://ssrn.com/abstract=2781104

- Einstein, Albert (1953), Preface, in: Galileo Galilei, Dialogue Concerning the Two Chief World Systems, übers. von Stillman Drake, Berkeley

- Eisenstein, Sergej M. (1974), Wie ich Regisseur wurde, in: Ders., Schriften, hg. von H. J. Schlegel, Bd. 1, Minden, 185-195

- Eisenstein, Sergej M. (2006), Montage der Attraktionen (1923), in: Ders., Jenseits der Einstellung. Schriften zur Filmtheorie, hg. vonFelix Lenz, Helmut H. Diederichs, Frankfurt am Main, 9-14

- Eisler, Rudolf, Karl Roretz (1930), Wörterbuch der philosophischen Begriffe, Bd. 3, 4. Aufl., Berlin

- Engbers, Jan (2001), Der »Moral-Sense« bei Gellert, Lessing und Wieland. Zur Rezeption von Shaftesbury und Hutcheson in Deutschland, Heidelberg

- Ferrari, Jean (1998), Das Ideal der reinen Vernunft, in: Mohr, Georg, Marcus Willaschek (Hg.), Immanuel Kant. Kritik der reinen Vernunft, Berlin, 491-523

- Gentner Dedre, Jonathan Grudin (1985), The Evolution of Mental Metaphors in Psychology: A 90-Year Retrospective, in: American Psychologist 40, 181-192

- Gombrich, Ernst (1973), Wertmetaphern in der bildenden Kunst, in: Ders., Meditationen über ein Steckenpferd. Von den Wurzeln und Grenzen der Kunst, Wien, 31-56

- Guillaume, Jean (1974), Léonard de Vinci et l'architecture française: I. Le problème de Chambord; II. La villa de Charles d'Amboise et le château de Romorantin; réflexion sur un livre de Carlo Pedretti, in: Revue de l'Art 25, 71-90

- Guillaume, Jean (2009), Léonard architecte de François Ier, in: Pedretti, Carlo (Hg.), Léonard de Vinci et la France, Foligno

- Hartmann, Nicolai (1949), Der Aufbau der realen Welt. Grundriss der allgemeinen Kategorienlehre, 2. Aufl., Meisenheim am Glan

- Heisenberg, Werner (1985), Die Bedeutung des Schönen in der exakten Naturwissenschaft, in: Ders., Gesammelte Werke, Abt. C, Bd. 3, Berlin, 369-384

- Herder, Johann Gottfried (1955), Kalligone, Weimar

- Heydenreich, Ludwig H. (1952), Leonardo da Vinci. Architect of Francis I., in: The Burlington Magazine XCIV, 277-283

- Höffe, Otfried (2011), Kants Kritik der reinen Vernunft. Die Grundlegung der modernen Philosophie, München

- Höffe, Otfried (2012), Bild - Metapher - Modell. Eine philosophische Einführung mit einigen Exempla, in: DERs. (Hg.), Bild und Bildlichkeit, Nova Acta Leopoldina, N. F. 113, Nr. 386, Halle / Saale, 9-21

- Hofstadter, Douglas, Emmanuel Sander (2014), Die Analogie. Das Herz des Denkens, Stuttgart

- Horn, Christoph (2005), Begriff, Ideen- und Wirkungsgeschichte, in: Depenheuer, Otto (Hg.), Staat und Schönheit. Möglichkeiten und Perspektive einer Staatskalokagathie, Wiesbaden, 23-32

- Jäckel, Michael (2006), Einführung in die Konsumsoziologie, Fragestellung - Kontroversen - Beispieltexte, 2. Aufl., Wiesbaden

- Jaeggi, Rahel (2014), Kritik der Lebensformen, Berlin

- Johnson, David J., Jessica Wortman, Felix Cheung, Megan Hein, Richard E. Lucas, M. Brent Donnellan, Charles R. Ebersole, Rachel K. Narr (2016), The Effects of Disgust on Moral Judgments. Testing Moderators, in: Social Psychological and Personality Science 7, 640-647

- Jürgens, Uta Maria, Danko Nicolić (2012), Ideaesthesia. Conceptual processes assign similar colours to similar shapes, in: Translational Neuroscience 3, 22-27

- Kant, Immanuel (1781), Kritik der reinen Vernunft, Riga

- Kant, Immanuel (1968), Kritik der Urteilskraft, in: Ders., Werke in zwölf Bänden, hg. von Wilhelm Weischedel, Band 10, Frankfurt am Main

- Kimmich, Dorothee (2011), Lebendige Dinge in der Moderne, Paderborn

- Kohl, Karl-Heinz (2003), Die Macht der Dinge. Geschichte und Theorie sakraler Objekte, München

- König, Gudrun M. (2009), Konsumkultur. Inszenierte Warenwelt um 1900, Wien, Köln, Weimar

- Koschorke, Albert (2012), Wahrheit und Empfindung. Grundzüge einer allgemeinen Erzähltheorie, Frankfurt am Main

- Kövecses, Zoltán (2010), Metaphor. A Practical Introduction, 2. Aufl., Oxford

- Kurz, Gerhard (2015), Das Wahre, Schöne, Gute. Aufstieg, Fall und Fortbestehen einer Trias, Paderborn

- Lakoff, George, Mark Johnson (2008), Leben in Metaphern. Konstruktion und Gebrauch von Sprachbildern, übers. von Astrid Hildenbrand, 6. Aufl., Heidelberg

- Landau, Mark J., Michael D. Robinson, Brian P. Meier (Hg.) (2014), The Power of Metaphor. Examining Its Influence on Social Life, Washington D.C.

- Landy, Justin F., Geoffrey P. Goodwin (2015), Does incidental disgust amplify moral disgust? A meta-analytic review of experimental evidence, in: Perspectives on Psychological Science 10, 518-536

- Langlois, Judith H., Lisa Kalakanis, Adam J. Rubenstein, Andrea Larson, Monica Hallam, Monica Smoot (2000), Maxims or Myths of Beauty? A Meta-Analytic and Theoretical Review, in: Psychological Bulletin 126, 390-423

- Lee, Spike W.S., Norbert Schwarz (2011), Wiping the Slate Clean. Psychological Consequences of Physical Cleansing, in: Current Directions in Psychological Sciences 20, 307-311

- Lepsius, Oliver (1999), Steuerungsdiskussion, Systemtheorie und Parlamentarismuskritik, Tübingen

- Levitan, Carmel A. et al. (2014), Cross-Cultural Color-Odor Associations, in: PLOS ONE 9, e101651

- Liesen, Pauline (1999), Architektur und Zeremoniell in den Schlössern Franz' I., Diss. Bonn

- Luhmann, Niklas (1993), Das Recht der Gesellschaft, Frankfurt am Main

- Luhmann, Niklas (1997), Die Gesellschaft der Gesellschaft, Berlin 
- Lynd, Robert S., Helen Merrell Lynd (1937), Middletown in Transition. A Study in Cultural Conflicts, New York, 242-294

- Martino, Gail, Lawrence G. Marks (2001), Synesthesia. Strong and weak, in: Current Directions in Psychological Science 10, 61-65

- McAllister, James W. (1996), Beauty and Revolution in Science, Ithaca, London

- McGlone, Matthew S., Jessica Tofighbakhsh (2000), Birds of Feather Flock Conjointly (?). Rhyme as Reason in Aphorisms, in: Psychological Science 11, 424-428

- Meier, Brian P., Michael D. Robinson (2004), Why the Sunny Side is Up. Associations Between Affect and Vertical Positions, in: Psychological Science 15, 243-247

- Meier, Christian (2000), Politik und Anmut. Eine wenig zeitgemäße Betrachtung, Stuttgart, Leipzig

- Metternich, Wolfgang (1985), Schloss Chambord an der Loire: der Bau von 1519-1524, Darmstadt

- Miller, Daniel (2010a), Der Trost der Dinge. Fünfzehn Porträts aus dem London von heute, Berlin

- Miller, Daniel (2010b), Stuff, Cambridge

- Möbius, Hanno (2000), Montage und Collage. Literatur, bildende Künste, Film, Fotografie, Musik, Theater bis 1933, München

- Möllers, Christoph (2015), Die Möglichkeit der Normen. Über eine Praxis jenseits von Moralität und Kausalität, Berlin

- Mroczko-Wąsowicz, Aleksandra, Danko Nikolić (2014), Semantic mechanisms may be responsible for developing synaesthesia, in: Frontiers in Human Neuroscience 8, 509, DOI: 10.3389, fnhum.2014.00509

- Nachtigall, Werner (1997), Vorbild Natur. Bionik-Design für funktionelles Gestalten, Berlin

- Nietzsche, Friedrich (1980), Sämtliche Werke. Kritische Studienausgabe, hg. von Giorgio Colli, Mazziano Montinari, München

- Nietzsche, Friedrich (1988), Die fröhliche Wissenschaft, in: Ders., Sämtliche Werke. Kritische Studienausgabe, hg. von Giorgio Colli, Mazzino Montinari, Bd. 3, 2. Aufl., München

- Ong, How Hwee, O’Dhaniel A. Mullette-Gillman, Kenneth Kwok, Julian Lim (2014), Moral judgment modulation by disgust is bi-directionally moderated by individual sensitivity, in: Frontiers in Psychology 5, art. 194

- Open Science Collaboration (2015), Estimating the reproducibility of psychological science, in: Science 349, aac4716

- Palladio, Andrea (1983), Die vier Bücher zur Architektur, nach der Ausgabe Venedig 1570 aus dem Italienischen übertragen und herausgegeben von Andreas Beyer und Ulrich Schütte, Zürich, München

- Panofskr, Erwin (1954), Galileo as a Critic of the Arts, The Hague

- Papillon, T., SNS (1998), Art. »Kalokagathie«, in: Historisches Wörterbuch der Philosophie, Bd. 4, Basel, Sp. 861-864

- Parise, Cesare V. (2016), Crossmodal Correspondences. Standing Issues and Experimental Guidelines, in: Multisensory Research 29, 7-28, DOI: 10.1163/22134808-00002502

- Peil, Dietmar (1983), Untersuchungen zur Staats- und Herrschaftsmetaphorik in literarischen Zeugnissen von der Antike bis zur Gegenwart, München

- Peil, Dietmar (2002), Bildfelder in historischer Perspektive, in: Handbücher zur Sprach- und Kommunikationswissenschaft, Bd. 21: Lexikologie. Ein internationales Handbuch zur Natur und Struktur von Wörtern und Wortschätzen, hg. von David A. Cruse, Herbert Ernst Wiegand, Berlin, 764-770

- Piaget, Jean, Bärbel Inhelder (1996), Die Psychologie des Kindes, 6. Auf., München

- Platon (2011a), Werke, Bd. 4: Politeia, hg. von Gunther Eigler, übers. von Friedrich Schleiermacher, 6. Aufl., Darmstadt

- Platon (2011b), Werke, Bd. 8, 2: Nomoi, hg. von Gunther Eigler, übers. von Klaus Schöpsdau, Hieronymus Müller, 6. Aufl., Darmstadt

- Poincaré, Jules Henri (1920), Science et méthode, Paris

- Popp, Joseph (1915), Deutsches Warenbuch, Hellerau bei Dresden

- Prinz, Wolfram (1980), Schloss Chambord und die Villa Rotonda in Vicenza. Studien zur Ikonologie, Berlin

- Prinz, Wolfram, Ronald G. Kecks (1994), Das französische Schloss der Renaissance. Form und Bedeutung der Architektur, ihre geschichtlichen und gesellschaftlichen Grundlagen, 2. Aufl., Berlin

- Ramachandran, V.S., E. M. Hubbard (2001), Synaesthesia. A window into perception, thought and language, in: Journal of Consciousness Studies 8, 3-34

- Ramsey, Jennifer L., Judith H. Langlois (2002), Effects of the »Beauty Is Good» Stereotype on Children's Information Processing, in: Journal of Experimental Child Psychology 81, 320-340

- Reber, Rolf, Christian Unkelbach (2010), The Epistemic Status of Processing Fluency as Source for Judgment of Truth, in: Review of Philosophy and Psychology 1, 563-581

- Reber, Rolf, Morten Brun, Karoline Mittendorfer (2008), The use of heuristics in intuitive mathematical judgment, in: Psychonomic Bulletin and Review 15, 1174-1178

- Reber, Rolf, Norbert Schwarz, Piotr Winkielman (2004), Processing Fluency and Aesthetic Pleasure. Is Beauty in the Perceiver's Experience?, in: Personality and Social Psychology 8, 364-382

- Rhode, Deborah L. (2010), The Beauty Bias. The Injustice of Appearance in Life and Law, Oxford

- Ryle, Gilbert (1949), The Concept of Mind, London

- Schiller, Friedrich (2005), Die Künstler, in: Ders., Sämtliche Werke (Berliner Ausgabe), Bd. 1: Gedichte, bearb. von JocheN GoLz, Berlin, 201-214

- Schnall, Simone, Jennifer Benton, Sophie Harvey (2008), With a Clean Conscience. Cleanliness Reduces the Severity of Moral Judgments, in: Psychological Science 19, 1219-1222

- Schnall, Simone, Jonathan Haidt, Gerald L. Clore, Alexander H. Jordan (2008), Disgust as Embodied Moral Judgment, in: Personality and Social Psychology Bulletin 34, 1096-1109

- Schnall, Simone, Jonathan Haidt, Gerald L. Clore, Alexander H. Jordan (2015), Landy and Goodwin (2015) Confirmed Most of Our Findings Then Drew the Wrong Conclusions, in: Perspectives on Psychological Science 10, $537 \mathrm{f}$. 
- Schwarz, Norbert, Fritz Strack (2014), Does Merely Going Through the Same Moves Make for a »Direct« Replication? Concepts, Contexts, and Operationalizations, in: Social Psychology 45, $305 \mathrm{f}$.

- Selle, Gert (2007), Geschichte des Design in Deutschland, 2. Aufl., Frankfurt am Main, New York

- Simmel, Georg (2006), Die Großstädte und das Geistesleben, Frankfurt am Main

- Simner, Julia, Catherine Mulvenna, Noam Sagiv, Elias Tsakanikos, Sarah A. Witherby, Christine Fraser, Kirsten Scott, Jamie Ward (2006), Synaesthesia. The prevalence of atypical cross-modal experiences, in: Perception 35, 1024-1033

- Spence, Charles (2011), Crossmodal correspondences. A tutorial review, in: Attention, Perception \& Psychophysics 73 , 971-995

- Spence, Charles, Ophelia Deroy (2013a), How automatic are crossmodal correspondences?, in: Consciousness and Cognition $22,245-260$

- Spence, Charles, Ophelia Deroy (2013b), Why we are not all synesthetes (not even weakly so), in: Psychonomic Bulletin and Review 2, 643-664

- Stemmer, Peter (2008), Normativität. Eine ontologische Untersuchung, Berlin

- Stühler, Friedbert (2002), Alfred Döblin, Berlin Alexanderplatz, Wolfgang Koeppen, Tauben im Gras. Der moderne deutsche Großstadtroman, 2. Aufl., Hollfeld

- The Oxford English Dictionary (1989), hg. von J. A. Simpson, E. S. C. Weiner, Bd. 5, 2. Aufl., Oxford

- Thümmler, Sabine (1999), Die Werkstatt für Wandmalerei, in: Fiedler, Jeannine, Peter Feierabend, Bauhaus, Köln, $452-461$

- Todorov, Alexander, Chris P. Said, Andrew D. Engell, NikolaAs N. Oosterhof (2008), Understanding evaluation of faces on social dimensions, in: Trends in Cognitive Sciences 12, 455-460

- Todorov, Alexander, Sean G. Baron, Nikolaas N. Oosterhof (2008), Evaluating face trustworthiness: a model based approach, in: Social Cognitive and Affective Neuroscience 3, 119-127

- Triepel, Heinrich (1947), Vom Stil des Rechts. Beiträge zu einer Ästhetik des Rechts, Heidelberg

- Tsukiura, Takashi, Roberto Cabeza (2011), Shared brain activity for aesthetic and moral judgments: implications for the Beauty-is-Good stereotype, in: Social cognitive and affective neuroscience 6, 138-148

- Veblen, Thorstein (2007), Theorie der feinen Leute. Eine ökonomische Untersuchung der Institutionen, Frankfurt am Main

- Vec, Mıloš (2009), Multinormativität in der Rechtsgeschichte, in: Berlin-Brandenburgische Akademie der Wissenschaften (vormals Preußische Akademie der Wissenschaften), Jahrbuch 2008, Berlin, 155-166

- Vec, Miloš (2010), Die normative Struktur des decorum. Über den Einbruch der Mode in den Naturrechtsdiskurs der Aufklärung, in: Bayreuther, Rainer (Hg.), Musikalische Norm um 1700, Berlin, 183-201

- Vesting, Thomas (2015), Rechtstheorie, 2. Aufl., München

- Vesting, Thomas (2017), Die Fachgrenze als Kontaktzone. Plädoyer für eine kulturwissenschaftliche Öffnung der Rechtswissenschaft, in: Funke, Andreas, Konrad Lachmayer (Hg.), Formate der Rechtswissenschaft, Weilerswist, 237-254

- Vogenauer, Stefan (2015), Schlüsselworte in englischen Savigny-Übersetzungen, in: Duve, Thomas, Joachim Rückert (Hg.), Savigny international?, Frankfurt am Main, 251-344

- Wang, Tingting, Lei Mo, Ce Mo, Li Hai Tan, Jonathan S. Cant, Luojin Zhong, Gerald Cupchik (2015), Is moral beauty different from facial beauty? Evidence from an fMRI study, in: Scan 10, 814-823

- Ward, Janet (2001), Weimar Surfaces. Urban Visual Culture in 1920s Germany, Berkeley u. a.

- Watson, Alan (1974), Legal Transplants. An Approach to Comparative Law, Edinburgh

- Weinrich, Harald (1976), Münze und Wort. Untersuchungen an einem Bildfeld, in: Ders., Sprache in Texten, Stuttgart, 276-290

- Wheeler, Ladd, Youngmee Kim (1997), What is Beautiful is Culturally Good. The Physical Attractiveness Stereotype has Different Content in Collectivistic Cultures, in: Personality and Social Psychology Bulletin 23, 795-800

- Wokalek, Marie (2011), Die schöne Seele als Denkfigur. Zur Semantik von Gewissen und Geschmack bei Rousseau, Wieland, Schiller, Goethe, Göttingen

- Zhong, Chen-Bo, Brendon Strejcek, Niro Sivanathan (2010), A clean self can render harsh moral judgment, in: Journal of Experimental Social Psychology 46, 859-862 